Draft Version June 16, 2021

Preprint typeset using $\mathrm{IAT}_{\mathrm{E}} \mathrm{X}$ style emulateapj v. 5/2/11

\title{
CONSTRAINTS ON THE MAGELLANIC CLOUDS' INTERACTION FROM THE DISTRIBUTION OF OB STARS AND THE KINEMATICS OF GIANTS
}

\author{
Dana I. Casetti-Dinescu ${ }^{1}$, Katherine Vieira ${ }^{2}$, Terrence M. Girard ${ }^{1}$ and William F. van Altena ${ }^{1}$ \\ Draft version June 16, 2021
}

\begin{abstract}
Young, OB-type candidates are identified in a $\sim 7900 \mathrm{deg}^{2}$ region encompassing the Large and Small Magellanic Clouds (LMC/SMC) periphery, the Bridge, part of the Magellanic Stream (MS) and Leading Arm (LA). Selection is based on UV, optical and IR photometry from existing largearea surveys and proper motions from the Southern Proper Motion 4 catalog (SPM4). The spatial distribution of these young star candidates shows 1) a well-populated SMC wing which continues westward with two branches partially surrounding the SMC, 2) a rather narrow path from the SMC wing eastward toward the LMC which is offset by $1^{\circ}-2^{\circ}$ from the high-density $\mathrm{H}$ I ridge in the Bridge, 3) a well-populated periphery of the LMC dominated by clumps of stars at the ends of the LMC bar and 4) a few scattered candidates in the MS and two overdensities in the LA regions above and below the Galactic plane. Additionally, a proper-motion analysis is made of a radial-velocity selected sample of red giants and supergiants in the LMC, previously shown to be a kinematically and chemically distinct subgroup, most likely captured from the SMC. SPM4 proper motions of these stars also indicate they are distinct from the LMC population. The observational results presented here, combined with the known orbits of the Clouds, and other aspects of the LMC morphology, suggest an off-center, moderate to highly-inclined collision between the SMC and the LMC's disk that took place between 100 and 200 Myr ago.

Subject headings: Magellanic Clouds — Galaxy: halo — Galaxy: kinematics and dynamics — galaxies: interactions - stars: early-type
\end{abstract}

\section{INTRODUCTION}

The Magellanic Clouds (MC) offer an exemplary insight into galaxy interactions, which are known to be an important driver of galaxy evolution. This is due to their proximity to the Milky Way (MW) that allows for detailed mapping of their gaseous and stellar components, the 3D kinematics of their stellar component, and the chemical-abundance makeup of these components. The strongest clues to their interaction are the $\mathrm{H} I$ features known as the Magellanic Stream (MS), the Bridge, and the Leading Arm (LA) (e.g., Nidever et al. 2010 and references therein). Earlier work attributed the formation of these structures as being due to the tidal interaction between the Clouds and the MW, with the understanding that the SMC's tidal disruption produced the MS and LA (e.g., Gardiner \& Noguchi 1996, Connors et al. 2006). Another scenario for the origin of the MS is ram-pressure stripping of the LMC gas due to the motion of the Cloud through the gas in the Galactic halo (Mastropietro et al. 2005); this model however can not explain the LA. This earlier work was based on proper-motion measurements of small absolute value and the MW halo being modeled primarily as an isothermal sphere, which led to bound orbits of the Clouds around the MW.

More recent work offers a somewhat different picture. The HST proper-motion measurements of the Clouds by Kallivayalil et al. (2006a,b, hereafter K06, see also Piatek et al. 2008) indicate that the Clouds are on their

\footnotetext{
${ }^{1}$ Astronomy Department, Yale University, P.O. Box 208101, New Haven, CT 06520-8101, USA, dana.casetti@yale.edu, terry.girard@yale.edu, william.vanaltena@yale.edu

2 Centro de Investigaciones de Astronomia, Apartado Postal 264, Mérida, 5101-A, Venezuela, kvieira@cida.ve
}

first passage (Besla et al. 2007, 2010) about the MW. The MS and LA are made of material pulled out from the SMC as in older models, however, the interaction is due solely to the LMC, far from the gravitational influence of the Galaxy (Besla et al. 2010). A more recent proper-motion study of the Clouds encompassing a 450 $\mathrm{deg}^{2}$ area was presented by Vieira et al. (2010, hereafter V10) based on Southern Proper Motion material (e.g., Girard et al. 2011). V10 were able to determine the most precise relative motion between the Clouds due to the global solution performed on the contiguous area encompassing the Clouds; this will help better constrain the recent interaction between the Clouds. While the size of the proper motions for both Clouds are slightly smaller than those from K06 (i.e., less energetic orbits) the results agree within formal uncertainties. See also the Costa et al. (2009) ground-based CCD measurements, which advocate smaller absolute value proper motions and the discussion about the accuracy of such measurements in Diaz \& Bekki (2012, hereafter DB12).

Thus, the new picture that the Clouds are on a more energetic orbit than earlier work had implied, appears real. However, there is still room for debate regarding the first-passage scenario proposed by Besla et al. (2007) or a two MW-pericentric passage scenario proposed by DB12 who explored a large orbital parameter space centered on the V10 proper motions and realistic MW halo potentials. The consensus is that the MS, LA and Bridge are of tidal origin, and formed from material pulled out from the SMC in its interaction with the LMC and perhaps the MW. The Galaxy's gravitational influence can help explain the bifurcation (Putman et al. 2003) of the MS, as proposed by DB12, and also suggested by the 
earlier work of Connors et al. (2006). In the Besla et al. (2010) model, no such bifurcation is seen. Moreover, the Besla et al. (2010) model implies that the Clouds will have a close encounter in the near future rather than in the near past, as indicated by many other observations.

The main criticism for tidal models is the absence of older stars in the MS and Bridge. Only young, blue stars were known to exist in the Bridge from the early work of Irwin et al. (1990), with the inference being that they were born in situ. Attempts to find older stars in the Bridge and MS as tidally stripped material have failed. However, there are recent indications that older stars in the Bridge do exist (Monelli et al. 2011), and perhaps previous searches of these stars were not wide enough and not necessarily in the right place (DB12). Another piece of evidence that tidal models are indeed on a sure footing is the study of Olsen et al. (2011, hereafter O11) who isolated a population of red giants, supergiants and Carbon stars in the LMC with peculiar kinematics. Their radial-velocity analysis indicated that these stars are either counter-rotating in a plane nearly coplanar with the LMC's disk, or have prograde rotation in a disk inclined by $\sim 54^{\circ}$ with respect to the LMC's disk. They also determined the abundances of 30 such stars as well as bona fide LMC stars. They found that the kinematically distinct stars have lower metallicities than do LMC stars; specifically, $[\mathrm{Fe} / \mathrm{H}]=-1.25 \pm 0.13$ dex, while LMC stars have $[\mathrm{Fe} / \mathrm{H}]=-0.56 \pm 0.02$ dex. The kinematical and chemical properties of these stars lead O11 to suggest that they were captured from the SMC.

In this study, we focus on two observational aspects that will help better model and understand the $\mathrm{LMC} / \mathrm{SMC} / \mathrm{MW}$ interaction. The first is to provide a reliable map of young, OB-type candidate stars over a $\sim 7900 \mathrm{deg}^{2}$ area that includes the Clouds' periphery, the Bridge, the LA and part of the MS. We select these candidates using photometry from the Galaxy Evolution Explorer survey (GALEX), the Two Micron All Sky Survey (2MASS), the Southern Proper Motion Program 4 (SPM4), and the American Association of Variable Star Observers All Sky Photometric Survey (APASS) and proper motions from SPM4. The second aspect, is a proper-motion analysis of the kinematically distinct $\mathrm{O} 11$ giants, in the LMC. In this study we have focused on OBtype candidates and giants in order to be able to reliably eliminate foreground contamination, and to have distant stars with apparent magnitudes in the range where SPM4 proper-motion errors are well under control.

The outline of the paper is as follows: in the next Section we present the catalogs used, followed by the selection of OB candidates in Section 3. In Section 4, we present the spatial distribution of the candidates in an area focused on the Clouds, then on a larger-scale area encompassing the MS and LA. In Section 5, we analyze the proper motions of the O11 sample and show that the proper-motions also indicate a kinematically distinct group reinforcing the conclusions of $\mathrm{O} 11$ and further characterizing the kinematical nature of this population. In Section 6 we discuss our findings in the context of the dynamical interaction of the Clouds and we summarize our results in Section 7 .

\subsection{The Southern Proper-Motion Catalog}

The fourth installment of the Yale/San Juan Southern Proper Motion Catalog, SPM4 contains positions, absolute proper motions and $B, V$ photometry for over 103 million objects south of $\delta=-20^{\circ}$. The catalog construction is described in Girard et al. (2011); it is roughly complete to $V=17.5$, and the proper-motion precision is 2-3 mas $\mathrm{yr}^{-1}$ for well-measured stars. At the bright end, proper motions are on the International Celestial Reference System via Hipparcos stars, while the faint end is tied to the inertial system via galaxies. About two thirds of the catalog include $V$ CCD photometry obtained with a $4 \mathrm{~K} \times 4 \mathrm{~K}$ PixelVision $(\mathrm{PV})$ camera $\left(0.83^{\prime \prime} / \mathrm{pix}\right)$ mounted in the focal plane of the yellow lens of the $50-\mathrm{cm}$ double astrograph at El Leoncito, Argentina. Initially, an Apogee $1 \mathrm{~K} \times 1 \mathrm{~K}$ camera $\left(1.3^{\prime \prime} / \mathrm{pix}\right)$ was placed behind the blue lens of the astrograph; this was later replaced by a $2 \mathrm{~K} \times 2 \mathrm{~K}$ Apogee Alta camera $\left(0.74^{\prime \prime} / \mathrm{pix}\right)$. The PV data were used for astrometry and $V$ photometry. The data from the Apogee cameras were used to obtain $B$ photometry only. Since the $B$ CCD area coverage is only about $20 \%$ of the PV area coverage, we did not use $B$ photometry in the current study. The CCD data were calibrated using Tycho 2 $B V$ values corrected to the Johnson system. While SPM4 also includes photographic photometry, we chose not to use it here, as it is less reliable than the CCD data. Hereafter, when mentioning SPM4 $V$ data, we refer solely to the calibrated PV CCD data. Internal error estimates (Girard et al. 2011) indicate a $V$ precision between 0.02 and 0.04 mags.

To further assess errors in the SPM4 $V$ photometry from an external comparison, we make use of the OGLE3 $V$ photometry in the LMC $^{3}$ '(Udalski et al. 2008). Since the SPM survey uses a diffraction grating in front of the objective, and the area under consideration is dense, crowding will affect the SPM4 photometry. Therefore this comparison will not necessarily reflect the average photometric errors in SPM4, but rather an upper limit. A total of 111735 objects were matched between the two catalogs, within a $2^{\prime \prime}$ matching radius, and with $12 \leq V_{O G L E 3} \leq 18$. In Figure 1 we show the area coverage of OGLE3 and that of the intersection of OGLE3 and SPM4 $V$. Isodensity contours of M giants selected from 2MASS (see Section 2.4) are also shown. The coordinates are a gnomonic projection of Galactic longitude and latitude centered at $(l, b)=\left(290^{\circ},-40^{\circ}\right)$. Throughout this paper, the spatial distribution will be represented with these coordinates, unless otherwise noted. In Figure 2 we show $V$-magnitude differences as a function of $V_{O G L E 3}$, for every tenth data point. The red line shows a moving median of the differences. A vertical line at $V_{O G L E 3}=16.7$ indicates the limiting magnitude of our OB-star candidates. This shallow limit compared to SPM4's $V=17.5$ limit is due to the fact that in its construction, SPM4 used several input catalogs including 2MASS for the faint end (Girard et al. 2011). Thus, the shallow $V$ limit in SPM4 reflects the 2MASS limit for these blue objects, rather than the photographic limiting magnitude of the SPM plates. From Fig. 2, the scatter in the $V$-magnitude differences is $\sim 0.07 \mathrm{mag}$

3 ftp.astrouw.edu.pl/ogle/ogle3/maps/lmc
2. CATALOGS 


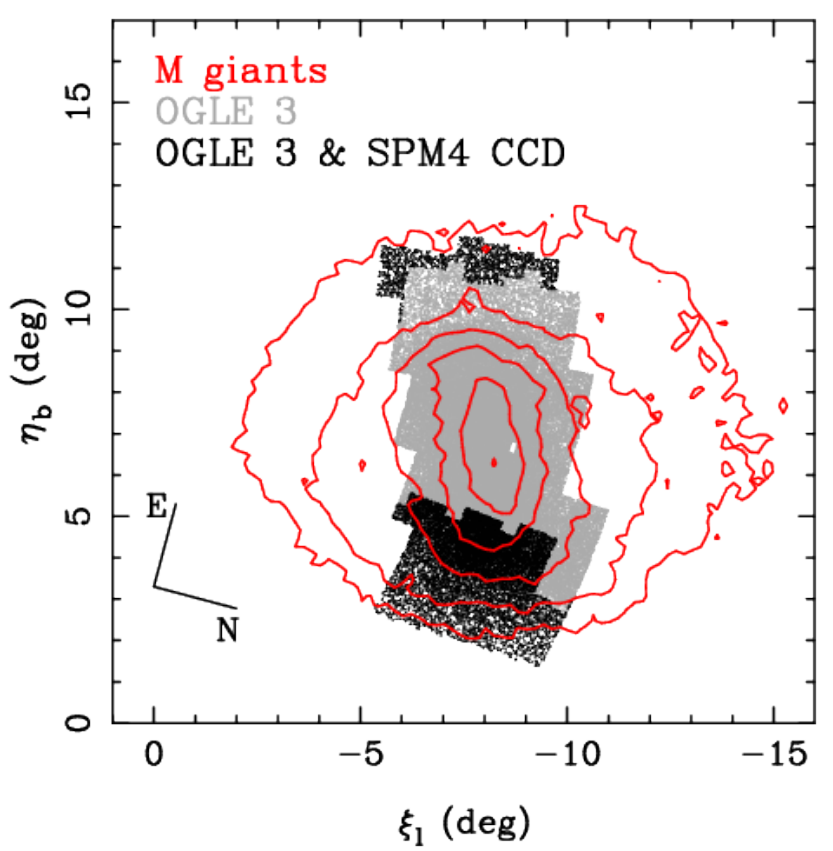

FIG. 1.- Spatial distribution of OGLE3 (grey) and the intersection of OGLE3 and SPM4 (black) in the LMC. M-giant isodensity contours are shown in red. Coordinates are a gnomonic projection of Galactic longitude and latitude centered at $(l, b)=\left(290^{\circ},-40^{\circ}\right)$.

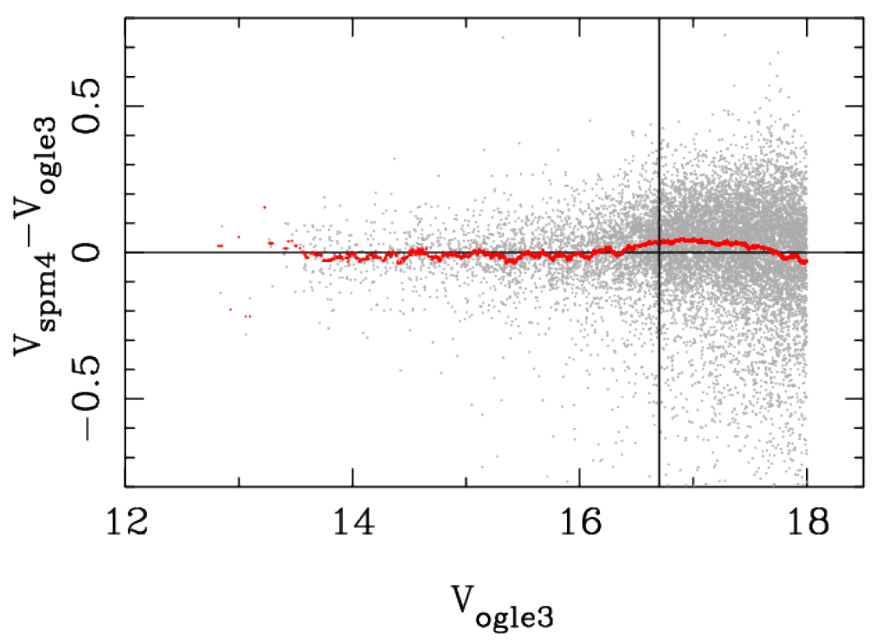

FIG. 2.- $V$-magnitude differences between SPM4 and OGLE3 as a function of $V_{O G L E 3}$. A moving median is shown in red; the vertical line indicates the limiting magnitude of our OB candidates.

for $13 \leq V \leq 16$ and $0.09 \mathrm{mag}$ for $16 \leq V \leq 17$. Since the dominant error in the differences is from SPM4 $V$, we will adopt these values as upper limits of the photometric errors in SPM4. The difference between SPM4 and OGLE3 as given by the moving median in $13.0 \leq V_{O G L E 3} \leq 16.5$ is $-0.025 \pm 0.001 \mathrm{mag}$.

Finally, we will also consider here a subcatalog built only in the area of the Clouds and described in V10. This catalog uses the same material as SPM4, but slightly different processing, with more careful treatment of magnitude-dependent systematics. The V10 catalog has $\sim 1.4$ million objects encompassing a 450- $\mathrm{deg}^{2}$ contiguous area including the Clouds and the Bridge (excluding the central regions of the Clouds due to crowding). It was built for the purpose of measuring the indi- vidual absolute proper motions of the Clouds and a very precise relative proper motion between the Clouds. We will also use the V10 proper motions in the selection of OB candidates to compare with the selection provided by SPM4 proper motions.

\subsection{The AAVSO All-Sky Photometric Survey}

To increase the area coverage of our study, we supplement the SPM4 $V$ data with $V$ photometry from the AAVSO All-Sky Photometric Survey - APASS4 described in Henden et al. (2011). Here we use the third data release, which includes Johnson $B, V$, Sloan $g, r, i$ and positions for 18.9 million objects. Magnitudes are reliable between $V \sim 10$ to 17.0 ; the pixel scale is $2.57^{\prime \prime}$ and the field of view is $2.9^{\circ} \times 2.9^{\circ}$. The catalog starts to be incomplete at $V \sim 16.0$; it is not yet fully contiguous, missing some $10 \%-20 \%$ in area.

Objects were matched by position, using a $2^{\prime \prime}$ matching radius. We compare $V$ magnitudes from SPM4 with those in APASS, in overlapping regions. For wellmeasured stars, magnitude differences have a scatter of $\sim 0.07-0.08 \mathrm{mag}$; assuming errors are similar in both catalogs, this indicates $V$ errors of $0.05-0.06$ mag. However, there are regions with significant offsets of the order of 0.5 to $1.0 \mathrm{mag}$; these regions have the size of the APASS field of view, therefore we believe they are some unreliably calibrated fields in APASS. We have carefully examined magnitude differences as a function of Declination and RA, and we find that large offsets are not present in the overall Clouds and Bridge areas; the largest offsets are present at $\delta<-80^{\circ}$. We therefore proceed to use APASS $V$ data for SPM4 objects that do not have CCD $V$ data. Of the entire list of objects to be examined $20 \%$ have $V$ from APASS; and of the list of OB candidates, $10 \%$ are from APASS.

\subsection{The GALEX UV Catalog}

Here, we use the GALEX fifth data release (GR5) catalog described by Bianchi et al. (2011). We focus on the all-sky imaging survey (AIS) that provides a list of 63.5 million unique sources with photometry in the far UV band $\left(F U V, 1344-1786 \AA, \lambda_{e f f}=1538.6 \AA\right)$ and the near UV band $\left(N U V, 1771-2831 \AA, \lambda_{e f f}=2315.7 \AA\right)^{5}$ Only sources with a distance $\leq 0.5^{\circ}$ from the field center and with errors $\epsilon_{N U V}<0.5 \mathrm{mag}$ were included in this catalog.

We match the GALEX sources with SPM4 objects by position using a matching radius of $3^{\prime \prime}$. Duplicate matches within this radius are eliminated by keeping the match with the smallest separation. In our analysis, we use only the $N U V$ band, since the $F U V$ band has far fewer detections.

\subsection{MASS and 6x2MASS}

The SPM4 catalog had been cross-matched with 2MASS (Skrutskie et al. 2006) prior to release (Girard et al. 2011); therefore $J, H, K$ magnitudes are present in the SPM4. In our analysis, we chose to use the $J$ band as it is the deepest IR band. Since our OB candidates

4 www.aavso.org/aavso-photometric-all-sky-survey-datarelease-1

5 archive.stsci.edu/prepds/bianchi-gr5xdr7/catalogUV.html 
have extremely blue colors and relatively faint $V$ magnitudes (see Section 3), the $J$ magnitudes are at the faint end of 2MASS, and thus prone to larger errors than the average of 2MASS. To verify that our OB candidate selection is not seriously affected by errors at the faint end of 2MASS, we also make use of the deeper survey $6 \mathrm{X}$ 2MASS in a few selected areas ${ }^{6}$. Specifically, we use the $383 \mathrm{deg}^{2}$ and $127 \mathrm{deg}^{2}$ areas in the LMC and SMC respectively. Thus, in addition to the 2MASS-based selection we present a $6 \mathrm{X}$ 2MASS-based selection for comparison.

For illustration purposes we construct isodensity contours of the M giants in the LMC and SMC; these represent an intermediately old population, of $[\mathrm{Fe} / \mathrm{H}] \sim-0.5$. $\mathrm{M}$ giants are selected from 2MASS within a $400-\mathrm{deg}^{2}$ box centered on the LMC, and a 225- $\mathrm{deg}^{2}$ box centered on the SMC. M giants are selected based on 2MASS colors according to the prescription described in Majewski et al. (2003).

\section{SELECTION OF OB-TYPE CANDIDATES}

We compile a list of about 4 million objects that have $N U V, V, J$ photometry and proper motions. For each object in this list we determine the reddening $\mathrm{E}_{B-V}$ from the Schlegel et al. (1998) maps and keep for further analysis objects with $E_{B-V}<0.5$. Objects are dereddened using the relationships from Majewski et al. (2003) for IR photometry, and those from Kinman et al. (2007) for the $N U V$ band. Specifically, $\mathrm{A}_{N U V}=8.90 \mathrm{E}_{B-V}$. For the $V$ band, we used $\mathrm{A}_{V}=3.1$. The Clouds are known to be affected by differential reddening due to the spatial variations of their intrinsic extinction as show by e.g., Zaritsky et al. (2004), Haschke et al. (2011). Our study in unlikely to be affected by this, as we sample regions in the outskirts of the Clouds where the extinction as well as its spatial variation is small according to Haschke et al. (2011).

In Figure 3 (top) we show the $(N U V-V)_{0}$ versus $(V-J)_{0}$ diagram, and the color-magnitude diagram (bottom). Grey symbols show our compiled list. The black curve shows model main-sequence colors, while the green curves are white-dwarf models taken from Vennes et al. (2010). The white-dwarf models correspond to two limiting masses of $0.4 \mathrm{M}_{\odot}$ and $1.2 \mathrm{M}_{\odot}$. We have also matched our list with catalogs of known-type objects, to check their color distribution. These are the QSO and AGN catalog by Véron-Cetty \& Véron (2006) (dark blue), RR Lyrae stars from the All Sky Automated Survey (ASAS) Szczygiel et al. (2009) (light blue), white dwarf candidates selected photometrically from GALEX and SDSS (Binachi et al. 2011) (green), and OB stars spectroscopically identified from Rolleston et al. (1999), Reed $(2003,2005)$, Bonanos et al. $(2009,2010)$ (in red). The initial color selection is shown as the black box with $-2.0 \leq(N U V-V)_{0} \leq 2.0$ and $-1.2 \leq(V-J)_{0} \leq-0.2$. We also trim by magnitude errors: $\epsilon_{N U V} \leq 0.10 \mathrm{mag}$ and $\epsilon_{J} \leq 0.15 \mathrm{mag}$. This selection implies that the latest stellar type is B8 according to the models of Vennes et al. (2010). The expected contamination in this color range is due to foreground stars such as OB subdwarfs and white dwarfs. Our selection provides 3099 objects of which only two are classified as non-stellar objects: one is a 2MASS extended source, the other a LEDA galaxy.

6 www.ipac.caltech.edu/2mass/releases/allsky/doc/

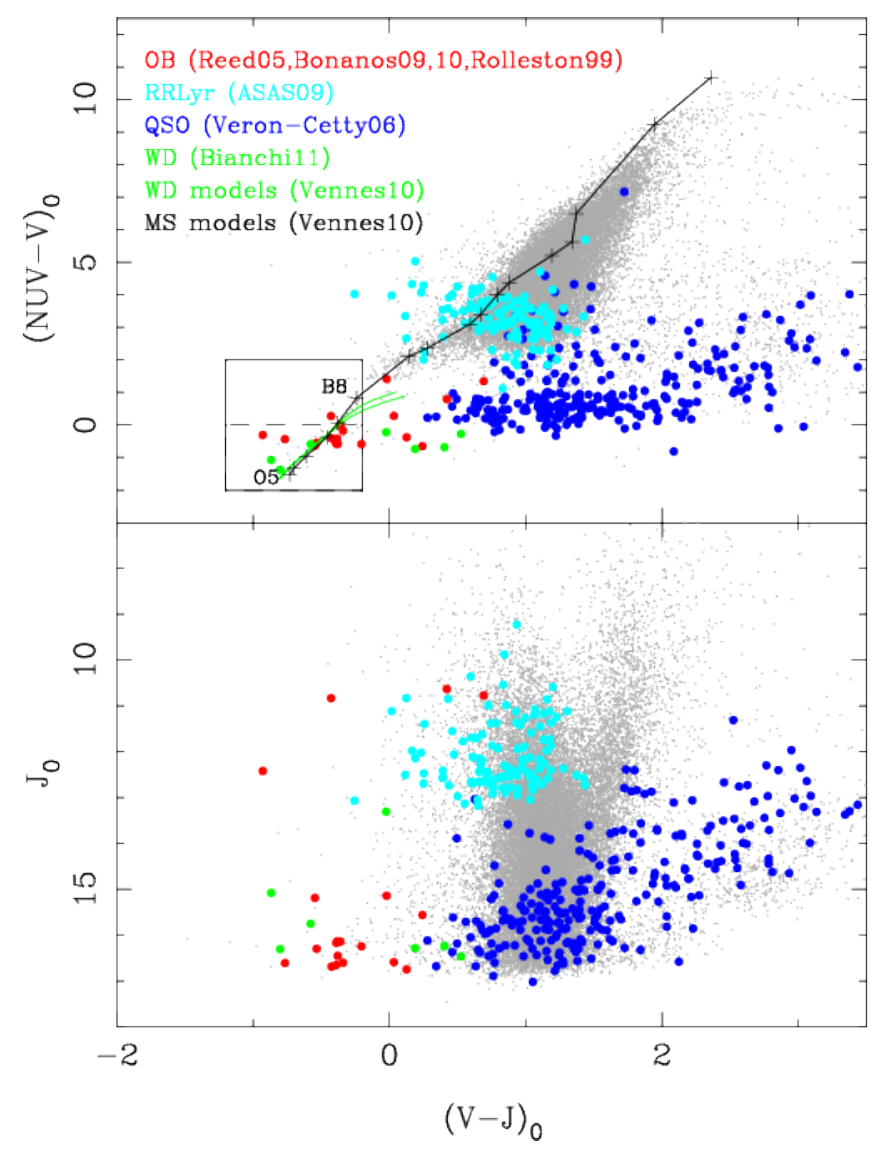

Fig. 3.- Color-color (top) and color-magnitude (bottom) plots of our list of objects with UV, optical and IR photometry and with proper motions (grey symbols). Main-sequence models are shown with a black line, and white-dwarf models with a green line. Objects of known type from various catalogs (see text) matched with our list are also shown and labeled. The preliminary color selection is represented by the black box; the more restrictive final color selection is represented with a dashed line.

In Figure 4 we show the spatial distribution in Galactic coordinates of this sample (top), and the $V_{0}$ distribution as a function of Galactic $l$ (middle) and $b$ (bottom). Between $l=-90^{\circ}$ to $-55^{\circ}$ and $b=-50^{\circ}$ to $-25^{\circ}$ one can see as a density enhancement the periphery of the LMC and SMC's wing. Density enhancements also appear at low latitudes, most likely representing disk populations. This is also apparent in the middle and bottom panels of Fig. 4; the Clouds' population sets in at $V_{0} \sim 13$. Therefore we keep only objects with $V_{0} \geq 13.0$. The faint limit of our color-selected sample is $V_{0} \sim 16.5$. Incompleteness begins in at $V_{0} \sim 15.5$, primarily due to 2MASS.

Next, we divide our color-selected sample into four $(N U V-V)_{0}$ subsamples and explore their spatial and proper-motion distributions. From Figure 5, the most prominent Clouds sample is in the color range $(-1.0<$ $\left.(N U V-V)_{0}<0.0\right)$, while the reddest subsample appears devoid of Clouds stars. From the proper-motion distributions shown in Figure 6, it is also clear that the color range $\left(-1.0<(N U V-V)_{0}<0.0\right)$ has a tight propermotion clump centered around $\left(\mu_{l}, \mu_{b}\right) \sim(-1.0,1.0)$ mas $\mathrm{yr}^{-1}$ and is indicative of a kinematically cold and distant population. We therefore choose a stringentlydefined sample by restricting the color range $(-2.0<$ $\left.(N U V-V)_{0}<0.0\right)$, i.e., selecting the bluest two sus- 

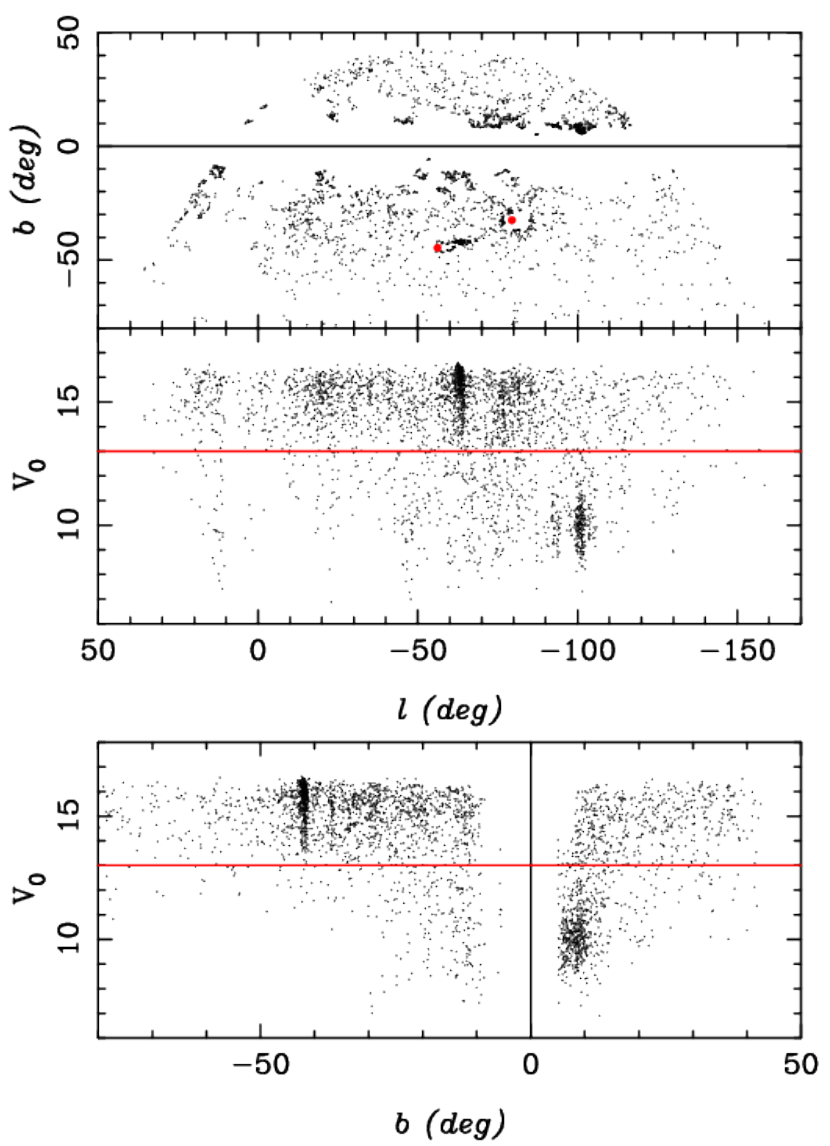

FIG. 4. - Spatial distribution of the color-selected sample $-2.0 \leq$ $(N U V-V)_{0} \leq 2.0$ and $-1.2 \leq(V-J)_{0} \leq-0.2$ (top), and $V$-magnitude distribution as a function of Galactic coordinates. The red line indicates the $V_{0}$ magnitude cut intended to eliminate nearby, foreground objects. The centers of the Clouds are marked with a red dot in the top panel.

bsamples shown in Figs. 5 and 6 . This will limit our sample to spectral types earlier than B5 $\left(T_{\text {eff }}>16,000\right.$ $\mathrm{K})$. By limiting to the bluest colors, we aim to target only the youngest distant stars, as well as to avoid some of the foreground contamination due to OB subdwarfs, which are known to be less frequent at bluer colors (e.g., Vennes et al. 2010). To eliminate nearby stars (i.e. white dwarfs) we also trim in proper-motion space as follows. We determine the average proper motion of stars located in the SMC wing, then we keep only stars within a proper-motion radius $\leq 8.0$ mas yr$^{-1}$ from this mean and with proper motion errors $\epsilon_{\mu_{l}} \leq 4.0$ and

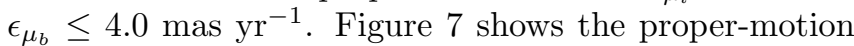
cut for the color and magnitude-selected sample (left), and the proper-motion distribution in each coordinate (right). There are 567 stars in this final sample, which was constructed to be as free as possible from foreground contamination. Some OB-type stars that are genuine members of the Clouds's system will be missed due to the circular footprint of GALEX, as well as due to the lack of photometry (mostly UV, but some $V$ as well, see next Section).

\section{SPATIAL DISTRIBUTION OF OB-TYPE CANDIDATES}

4.1. The Clouds and Bridge Region
In Figure 8 we show the gnomonic projection of the spatial distribution of our OB-type candidates, in a 2500$\operatorname{deg}^{2}$ region centered at $(l, b)=\left(290^{\circ},-40^{\circ}\right)$. Data combinations from various surveys are also highlighted in different colors as follows: GALEX and $V$ from SPM4 in green, GALEX and $V$ from APASS in yellow, GALEX and no $V$ data in orange. White gaps are due to the lack of GALEX data: note the round footprint of the GALEX catalog. 2MASS data and SPM4 proper motions are practically everywhere in this area, and thus not highlighted. The location of the Clouds is represented with M-giant isodensity contours from 2MASS (see also Section 2.4). Our OB-type candidates are shown with blue circles. We have also applied the same selection criteria, but replacing the $J$-magnitude from 2MASS, with that from 6X 2MASS, for $J>13.0$ mag. The resulting sample contains 586 stars, i.e. 19 more. The spatial distribution is essentially unchanged from that of the 2MASS-only sample. The 6X 2MASS sample is shown in Figure 9; the two clumps at the end of the LMC bar appear slightly more enhanced than in the 2MASS sample.

Finally, we perform one more test. We replace the SPM4 proper motions with the proper motions from the catalog of V10 based on the same material as SPM4, but with slightly different processing. We regard this latter catalog as more accurate and precise than the SPM4, since more careful treatment was possible in this restricted area. The V10 catalog covers a contiguous 450$\mathrm{deg}^{2}$ region encompassing the Clouds, and excluding the inner regions of the Clouds. In Figure 10 we show the same data from Fig. 8, only with the OB candidates being selected in proper-motion space using the V10 catalog and the same criteria as in the previous Section.

In both cases, the spatial distribution of $\mathrm{OB}$ candidates shows a very well-populated SMC wing, with two branches partly surrounding the SMC; the opposite side from the SMC wing, i.e., the north-west periphery of the SMC appears devoid of these stars. From the SMC wing a rather narrow $\left(\sim 3^{\circ}\right)$ band extends toward the LMC, ending some $\sim 4^{\circ}$ from the LMC periphery, or at RA $\sim 3.4^{h}$. A group of $2-3$ candidates is present in the periphery of the LMC and in the direction of the band coming from the SMC wing at $(l, b) \sim\left(286.3^{\circ},-36.9^{\circ}\right),(\mathrm{RA}$, Dec $)=\left(4.2^{h},-72.9^{\circ}\right)$; perhaps this indicates the spot where material from the SMC is funneled into the LMC after the close encounter between the Clouds. While there appears to be a gap of $\sim 4^{\circ}$ from the easternmost end of the SMC wing to the group of 2-3 stars in the LMC's periphery, it is also possible that we miss candidates due to the GALEX gaps (see Fig. 8).

The LMC periphery is well populated with these OB candidates, with some of them scattered at nearly $10^{\circ}$ from the LMC's center. Most remarkably, there are two clumps of these stars at each end of the bar, some $5^{\circ}$ from the LMC's center. In the north-west clump there are 19 stars (23 if we use $6 \times 2$ MASS), and in the southeast clump there are 8 stars (9 if we use $6 \times 2 \mathrm{MASS}$ ). The V10 proper-motion catalog excluded inner regions of the LMC, thus, these areas in the LMC are not well represented in V10. Also, a clump is seen in an armlike feature, where known young associations and clusters exist (see below). The northern side of the LMC (which roughly corresponds to the near side) appears to have a 

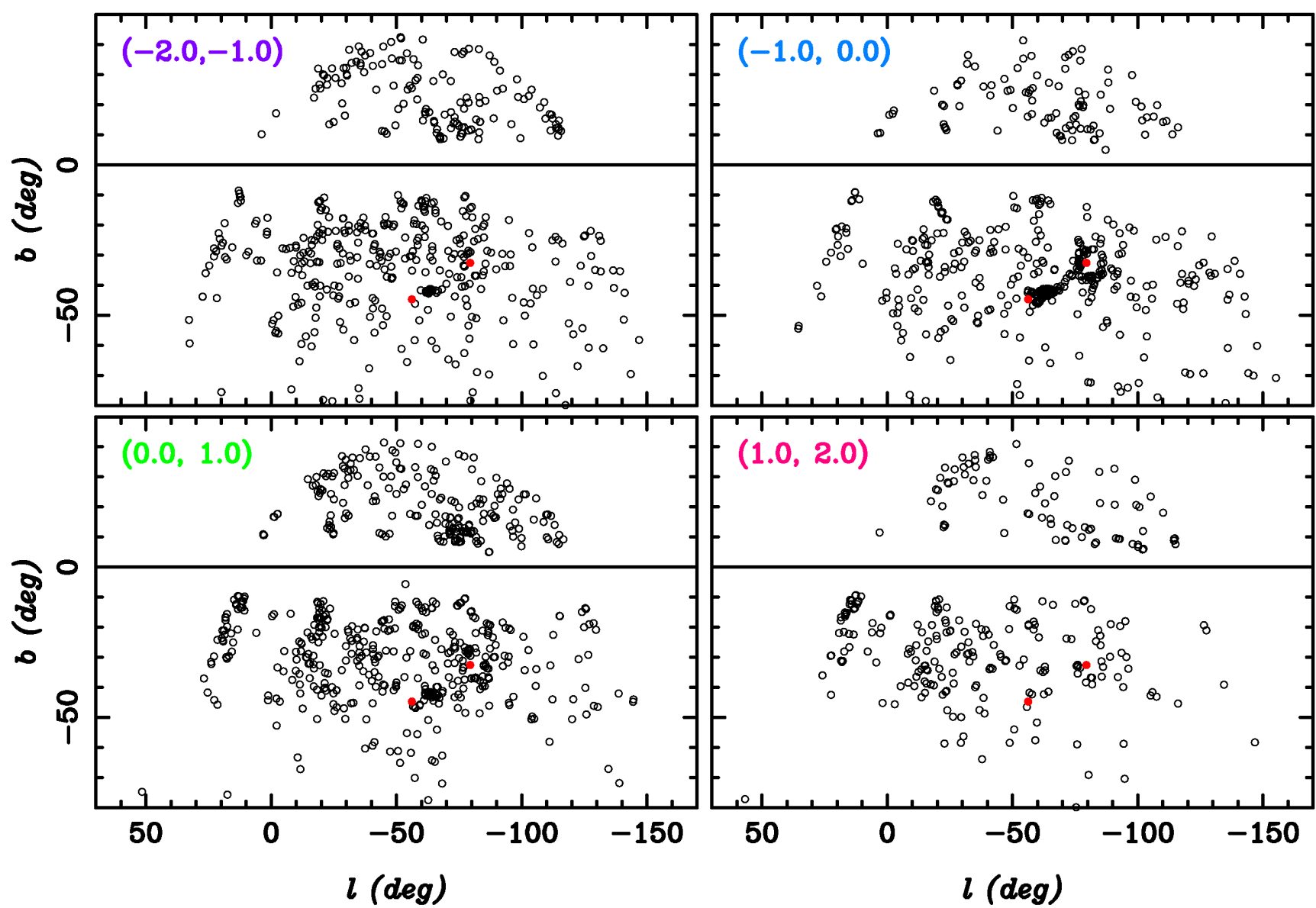

FIG. 5.- Galactic-coordinate distributions of four subsamples selected by $(N U V-V)_{0}$ color. The overall sample comes from the $-1.2 \leq(V-J)_{0} \leq-0.2, V_{0} \geq 13.0$ mag cuts. The $(N U V-V)_{0}$ interval is specified in each panel. The centers of the Clouds are marked with a red dot.
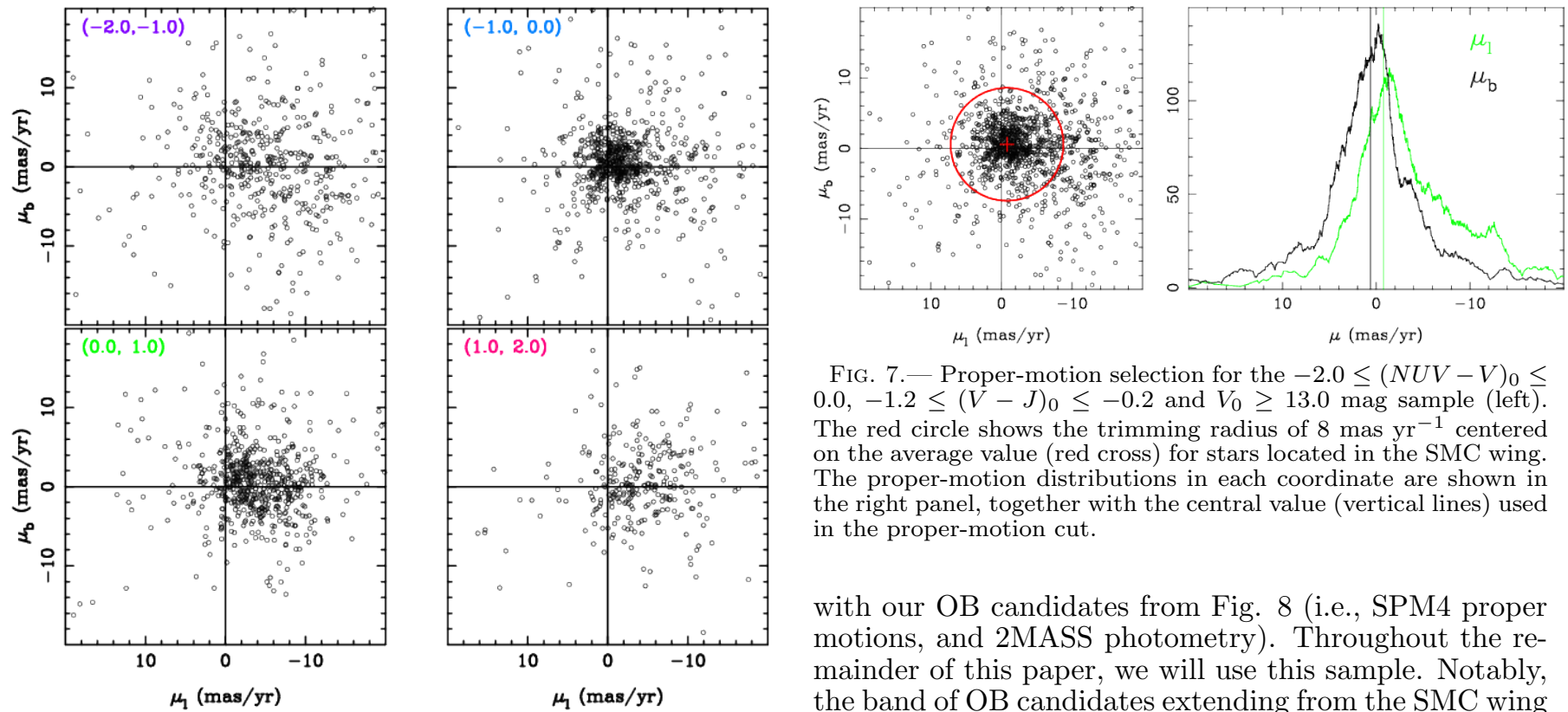

FIG. 7.- Proper-motion selection for the $-2.0 \leq(N U V-V)_{0} \leq$ $0.0,-1.2 \leq(V-J)_{0} \leq-0.2$ and $V_{0} \geq 13.0 \mathrm{mag}$ sample (left).

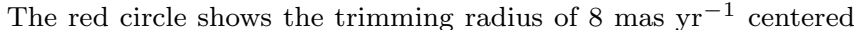
on the average value (red cross) for stars located in the SMC wing. The proper-motion distributions in each coordinate are shown in the right panel, together with the central value (vertical lines) used in the proper-motion cut.

with our OB candidates from Fig. 8 (i.e., SPM4 proper motions, and 2MASS photometry). Throughout the remainder of this paper, we will use this sample. Notably, the band of $\mathrm{OB}$ candidates extending from the $\mathrm{SMC}$ wing toward the $\mathrm{LMC}$ (i.e. at RA $>3^{h}$ ) is offset by $1^{\circ}-2^{\circ}$ from the highest $N_{H I}$ density region in the bridge shown with a white line in Fig. 11. This also holds for the group of three candidates in the periphery of the LMC, at $(l, b) \sim\left(286.3^{\circ},-36.9^{\circ}\right),(\mathrm{RA}, \mathrm{Dec})=\left(4.2^{h},-72.9^{\circ}\right)$.

Next, we compare our list of 567 candidates with the 


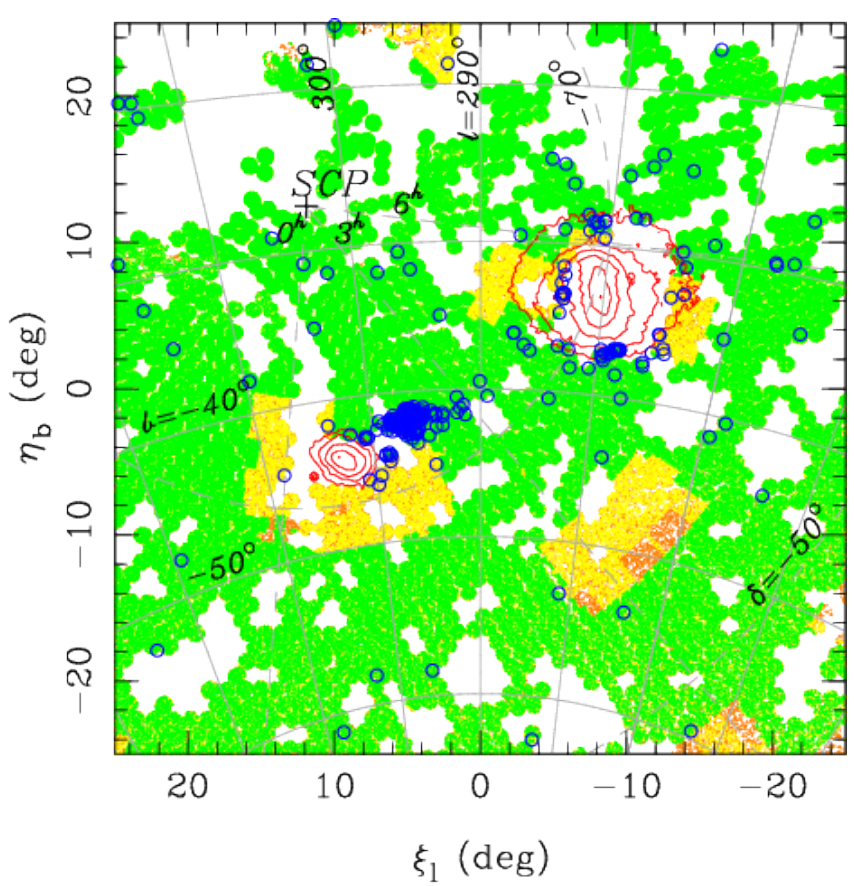

FIG. 8. - Spatial distribution of OB-type candidates (blue circles). Regions with GALEX and $V$ from SPM4 are shown in green, regions with GALEX and $V$ from APASS in yellow, and regions with only GALEX photometry are shown in orange. M-giant isodensity contours in the Clouds' areas are shown in red.

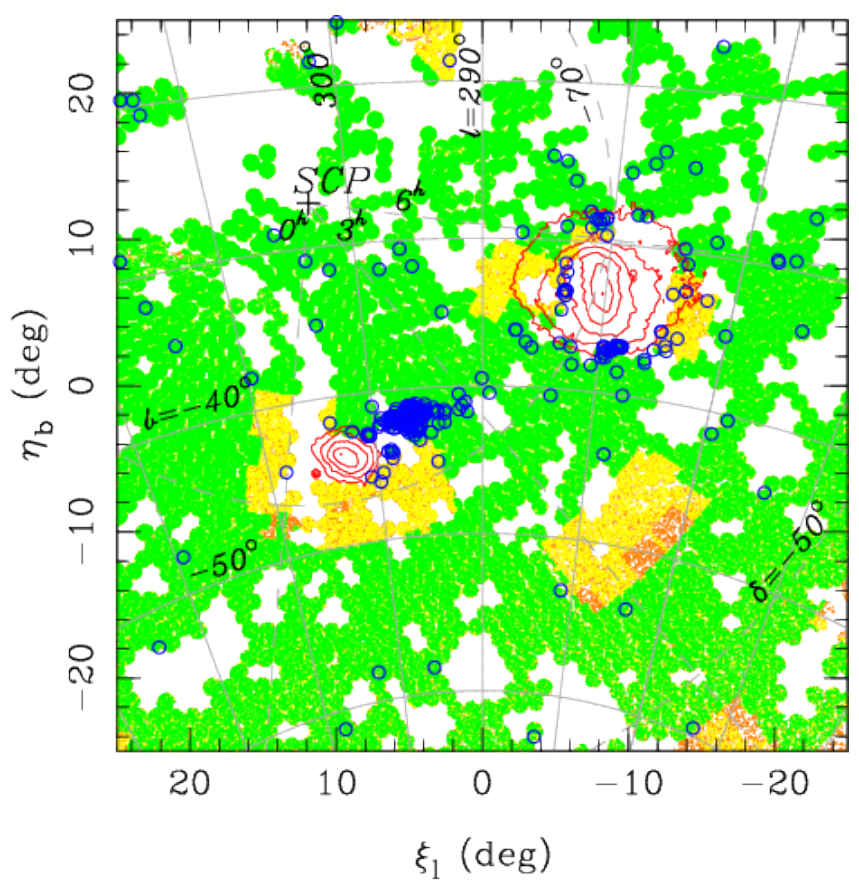

FIG. 9.- Same as Fig.8, except that the 2MASS data have been replaced with $6 \mathrm{X} 2 \mathrm{MASS}$ data for $J>13.0 \mathrm{mag}$ in making the selection of OB-type candidates.

Bica et al. (2008) catalogs of emission nebulae (1445 objects), stellar associations (3326) and clusters (3740) in the Clouds and Bridge. We search for positional coincidence among our $\mathrm{OB}$ candidate list and each of the Bica et al. (2008) catalogs; matched objects have separations that are smaller than or equal to the average radius of the extended object (i.e., $0.5 \times$ (semimajor+semiminor

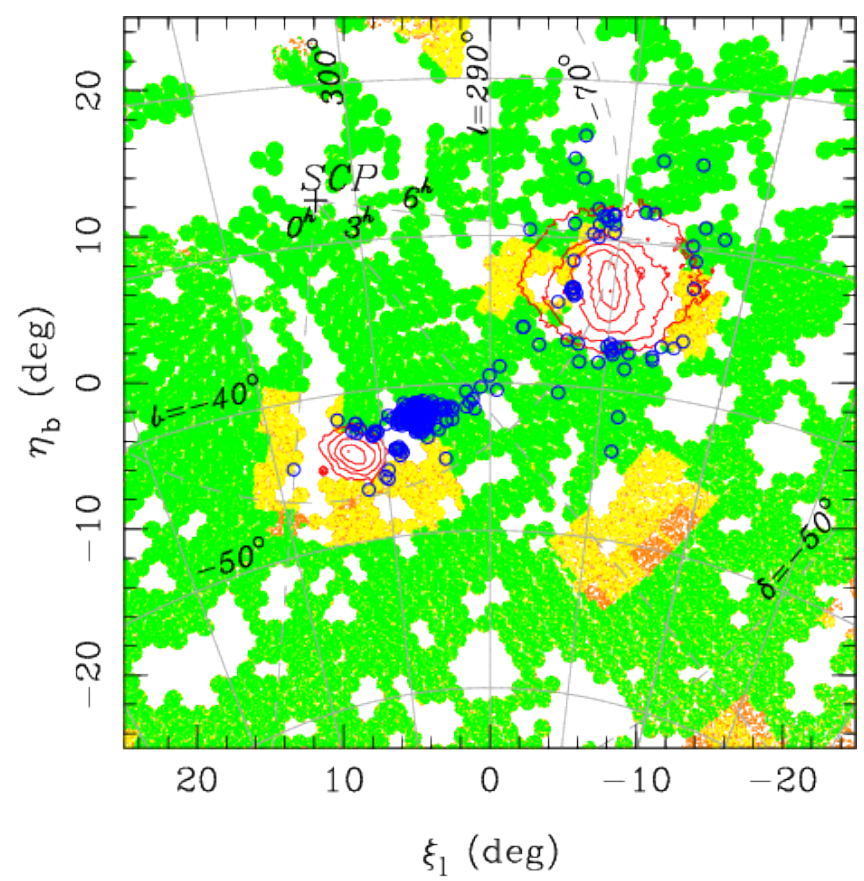

FIG. 10.- Same as Fig.8, except that the SPM4 proper motions have been replaced with the proper-motion catalog of V10, which covers a $450-\mathrm{deg}^{2}$ contiguous area encompassing the Clouds.

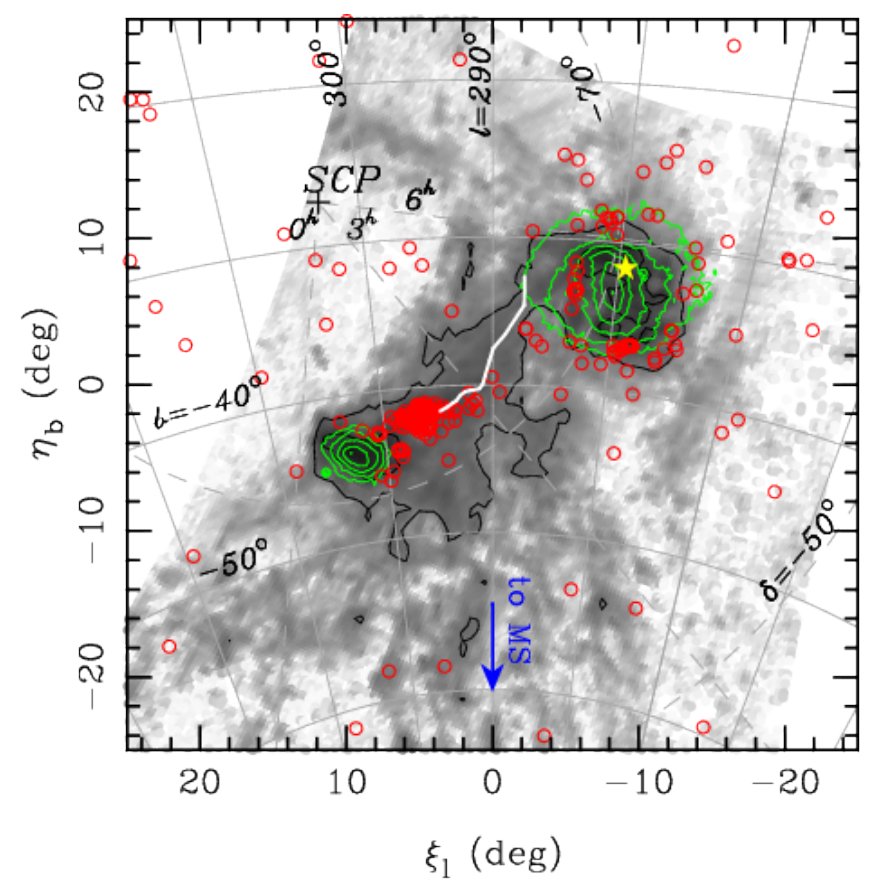

FIG. 11.- OB candidates (red circles) against the H I map from Putman et al. (2003) - grey scale. The black contours correspond to $N_{H I}=10^{20}$ and $10^{21}$ atoms $\mathrm{cm}^{-2}$; the latter one being barely visible in the inner regions of the LMC. M-giant isodensity contours are shown in green. The highest density region in the Bridge is highlighted with a ridge line shown in white. The 30 Doradus star-forming region is shown with a yellow star symbol.

axis) from Bica et al. 2008). Clusters in this catalog are those older than 5 Myr. We find 15 matches with the emission nebulae catalog, with 3 in the LMC, and the rest in the $\mathrm{SMC}$ wing; 71 matches with the associations catalog, with 2 in the SMC, 11 in the LMC and the rest 


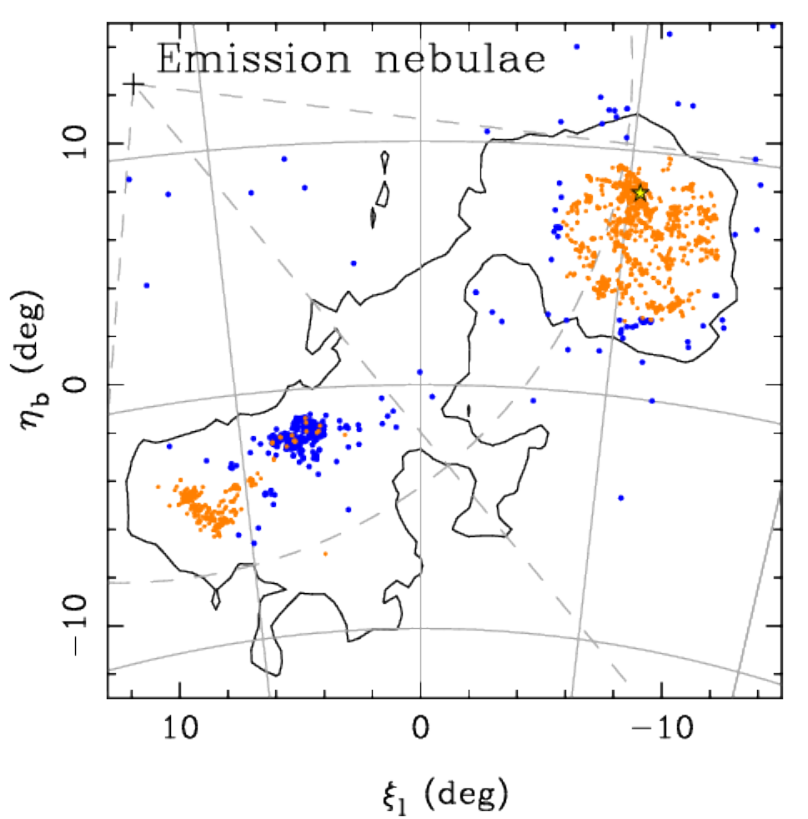

FIG. 12.- Distribution of emission nebulae from Bica et al. (2008) (orange), and our OB candidates (blue). The black contour represents the HI isodensity of $N_{H I}=10^{20}$ atoms $\mathrm{cm}^{-2} .30$ Doradus is shown with a yellow star symbol.

in the Bridge and SMC wing; and 10 matches with the cluster catalog, with 2 in the LMC, 1 near the edge of the $\mathrm{SMC}$ and the rest in the SMC wing. The distributions of each of these catalogs together with our OB candidates are shown in Figs 12, 13 and 14. Most of the matches are in the SMC wing/Bridge, and in the clump of stars to the left of the LMC bar, where clusters also show an enhancement resembling a spiral arm. The two clumps found at the ends of the LMC bar appear farther out than the extent of the emission nebulae and associations. In fact, none of the matches with all three catalogs are in these two clumps at the ends of the bar. In the SMC region, our candidates that form the two branches that partly surround the SMC do not seem to be the edges of a roundish distribution of associations/clusters within the SMC. Emission nebulae, and associations and even clusters in the SMC have an elongated distribution along the SMC bar, which is oriented nearly perpendicular to the direction to the SMC wing. The configuration seen in our $\mathrm{OB}$ candidates around the $\mathrm{SMC}$ is also apparent in the study of Gardiner \& Hatzidimitriou (1992) where blue stars were selected from the UKST photographic survey. Their Figure 5 indicates that the periphery (i.e., out to $\sim 2^{\circ}$ of the SMC center) is partly surrounded by blue stars; our study shows the same configuration is present out to radii of $3^{\circ}-4^{\circ}$.

To check possible differences in the population of $\mathrm{OB}$ candidates, we separate the Clouds region into a) an SMC region which includes the SMC wing and its extension to $\mathrm{RA} \sim 3.4^{h}$, and the branches surrounding the SMC, and b) the LMC region, which includes the periphery out to a radius of about $8^{\circ}$. We calculate the average $V_{0},\left(N U V_{V}\right)_{0}$, and $(V-J)_{0}$ and list these in Table 1. There is indication that the LMC sample is slightly brighter than the SMC one, and marginally bluer. The magnitude difference can easily be accounted for by the different distance moduli of the Clouds, i.e.,

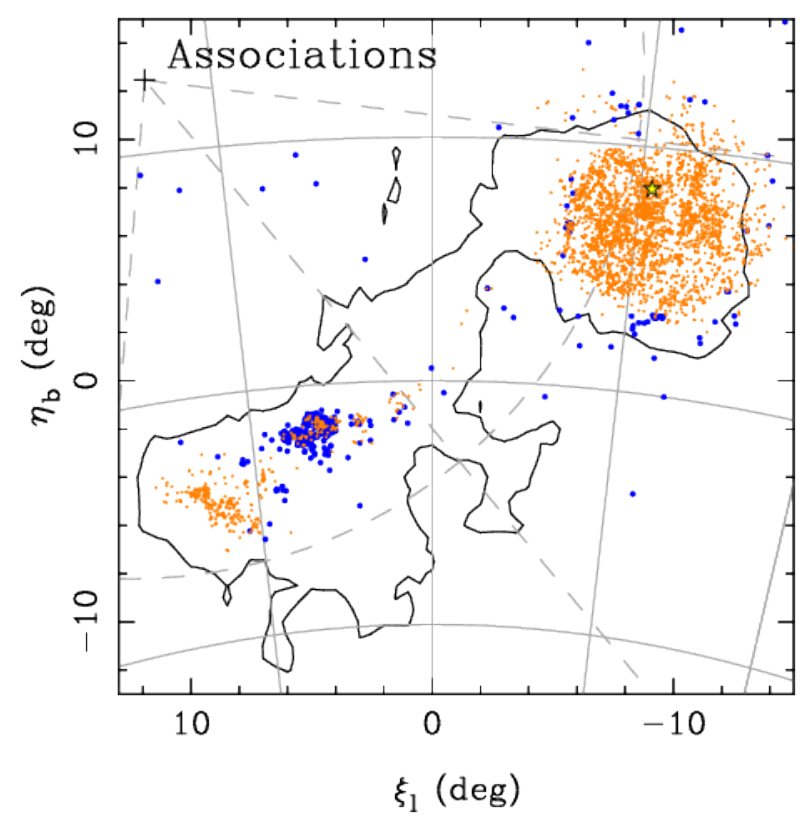

FIG. 13.- Same as in Fig. 12, but with stellar associations shown in orange.

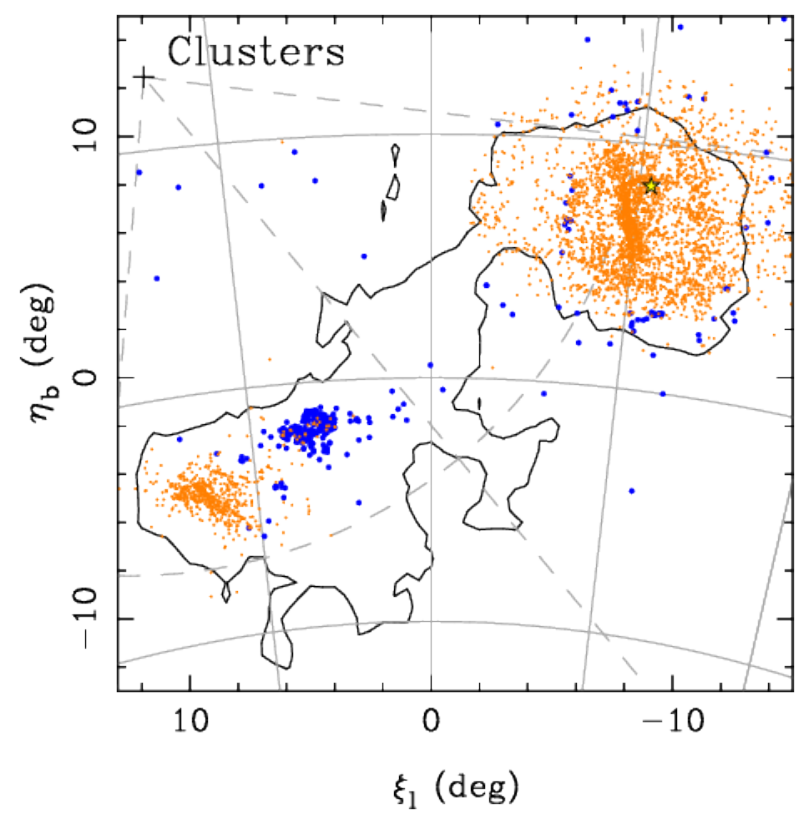

FIG. 14.- Same as in Fig. 12, but with clusters shown in orange.

$\Delta m=0.49$ if the distance to the LMC is $50.1 \mathrm{kpc}$ and to the SMC is $62.8 \mathrm{kpc}$ (van der Marel et al. 2002, hereafter vdM02). Thus, at a first look, there is no indication that the two groups consist of significantly different stellar populations.

TABLE 1

Average magnitudes AND COLORS

\begin{tabular}{lrr}
\hline & SMC region & LMC region \\
\hline$N$ & 270 & 71 \\
$<V_{0}>$ & $15.51 \pm 0.04$ & $15.08 \pm 0.09$ \\
$\left\langle(N U V-V)_{0}\right\rangle$ & $-0.62 \pm 0.02$ & $-0.70 \pm 0.04$ \\
$\left\langle(V-J)_{0}\right\rangle$ & $-0.48 \pm 0.01$ & $-0.58 \pm 0.02$ \\
\hline
\end{tabular}




\subsection{The Magellanic Stream and Leading Arm}

In Figure 15 we show the spatial distribution of our OB candidates in MS coordinates (e.g., Nidever et al. 2010). These are overplotted onto the H I column density map from Nidever et al. (2010) in the top panel, and onto our survey coverage in the bottom panel. There appear to be $\sim 10$ scattered candidates along the MS; only a few of these are in high $N_{H I}$ regions. There are $4-5$ candidates in the region opposite the SMC wing; these extend from $\Lambda_{M S}=-40^{\circ}$ to $-15^{\circ}$, and $B_{M S}=-15^{\circ}$ to $-28^{\circ}$. More prominent clumps of $\mathrm{OB}$ candidates can be found in the LA regions at $\left(\Lambda_{M S}, B_{M S}\right)=\left(16^{\circ},-20^{\circ}\right)$, below the Galactic plane, and at $\left(\Lambda_{M S}, B_{M S}\right)=\left(\sim 40^{\circ},-10^{\circ}\right.$ to $\left.0^{\circ}\right)$, above the Galactic plane. These clumps coincide with regions of moderate column density. Note however the gap in the survey for the clump below the Galactic plane: specifically the high $N_{H I}$ region at $\left(\Lambda_{M S}, B_{M S}\right)=$ $\left(15^{\circ},-25^{\circ}\right)$ is not covered by our data. Since these clumps are at low Galactic latitude, we may expect foreground contamination from stars in the MW disk. The clump below the plane is at $(l, b)=\left(298.2^{\circ},-12.2^{\circ}\right)$ and contains $\sim 10$ stars, while the grouping above the plane is at $(l, b)=\left(276.1^{\circ}-292.5^{\circ}, 11.7^{\circ}\right)$, and contains some 16 stars

The Galactic longitudes of these two groups encompass the Carina-Sagittarius arm and possibly the Perseus arm at large distances from the Sun (Vallée 2005, Carraro \& Costa 2009, Baume et al. 2009). Carraro \& Costa (2009) study the region at $l=290^{\circ}$ where they find three distinct groups of young stars: two of them at 2.5 and 6.0 $\mathrm{kpc}$ they believe belong to the Carina-Sagittarius arm as the line of sight crosses this arm twice, while the third at $\sim 12.7 \mathrm{kpc}$ is believed to belong to the Perseus arm. If we use these distances and the Galactic latitude of $|b| \sim 12^{\circ}$ of our two groups, we obtain distances from the Galactic plane of $0.5,1.2$ and $2.6 \mathrm{kpc}$ respectively. Thus, regardless of the stellar-population type, these stars, if postulated to be at the location of the spiral arms, appear rather far from the Galactic plane. One could invoke a warped disk to place material so far from the plane. However this explanation is also problematic, because the two clumps are below and above the plane at similar Galactic longitudes.

The most plausible contamination of our sample by foreground stars in this particular direction is from nearby stars (i.e., closer to the plane), possibly white dwarfs. On average, $V_{0}=14.6 \mathrm{mag}$ (with a range from $\sim 14$ to $16 \mathrm{mag}$ ) for our $\mathrm{OB}$ candidates in these two groups. Assuming $M_{V} \sim 10.0$ for white dwarfs (e.g., Vennes et al. 2010 models), we obtain a distance of the order of 100 pc. To check the white-dwarf frequency, we use the Besancon-model (Robin et al. 2003) predictions for the below-the-plane location of our clump of OB candidates. Specifically, in an area of $6 \mathrm{deg}^{2}$ we have 10 OB candidates. In the same area, the Besancon model predicts only one white dwarf that satisfies our color and magnitude criteria. However, its proper motion is $\sim 100$ mas $\mathrm{yr}^{-1}$, and therefore would not have made our propermotion cut.

We conclude that it is not reasonable to explain the density enhancements of OB candidates at low latitudes as spiral arm constituents, nor as foreground contamination from white dwarfs. Clearly, spectroscopic followup is necessary to elucidate this further. Nevertheless, our $\mathrm{OB}$ candidates suggest the existence of recently formed stars in the LA, an intriguing possibility that has bearing on its formation.

\section{PROPER MOTIONS OF THE OLSEN ET AL. (2011) SAMPLE}

\subsection{A Kinematically Distinct Population?}

Recently, O11 presented a radial-velocity study of a sample of $\sim 5900$ red supergiants, oxygen- and carbonrich asymptotic giant branch (AGB) stars and other giants. Examining the outliers from the fit of the LMC rotation curve, they find a population of stars that have radial velocities that are kinematically distinct. Based on radial velocities, O11 suggest these stars can either counter-rotate in a plane closely aligned with the LMC disk, or rotate in the same sense as the LMC disk, but in a plane inclined by $\sim 54^{\circ}$ to the LMC disk. This population represents $5 \%$ of the total population. O11 measure the metallicities of 30 such stars and find that they are also distinct, with $[\mathrm{Fe} / \mathrm{H}]=-1.25 \pm 0.13 \mathrm{dex}$ when compared to the bona fide LMC stars which have $[\mathrm{Fe} / \mathrm{H}]=-0.56 \pm 0.02$ dex. Their inference is that these stars were captured from the SMC, specifically from the periphery of the SMC.

Here, we use the O11 catalog matched with the SPM4 catalog to explore whether a proper-motion difference is present in the two samples. The majority of the O11 data lie in the inner regions of the LMC where propermotion measurements are most challenging due to crowding. We develop a differential approach to assess any proper-motion differences. We match 285 stars from the O11 "captured" sample (hereafter called captured-SMC) and 4138 from the "bona fide LMC" sample (hereafter called LMC) with SPM4, using a matching radius of $1.5^{\prime \prime}$. In Figure 16 we show the spatial distribution of the two samples in the same coordinate system as in Figs 8-10. For reference, the M-giant isodensity contours are shown in black. The LMC's center as adopted from vdM02 is also marked, as well as the line of nodes of the LMC disk with the orientation adopted from O11 (blue line).

To determine the proper-motion difference between the two samples, one can in principle take the average values for both samples; in practice, magnitude-dependent systematics that also vary with spatial location are likely to affect such a direct determination. For this reason, we develop a local, differential solution as follows. The LMC sample is chosen as a reference sample, while the captured-SMC as a target sample. For each target star, we define a local sample formed from $N_{l}$ neighboring, reference stars, and calculate the proper-motion difference between target and the average of the reference stars. Then, we take the average of these differences for all target stars after trimming outliers. We explore ranges for two parameters upon which such a solution might depend: $N_{l}=50$, and 200 and the proper-motion box size for trimming outliers in the target sample, i.e., \pm 20 mas $\mathrm{yr}^{-1}$ and \pm 40 mas $\mathrm{yr}^{-1}$ in both proper-motion coordinates. We also perform three types of local solutions which differ by the definition of the "distance" between reference and target stars. The first solution uses a metric that is a combination of spatial distance and $V$ magnitude difference. The spatial distance between target and 


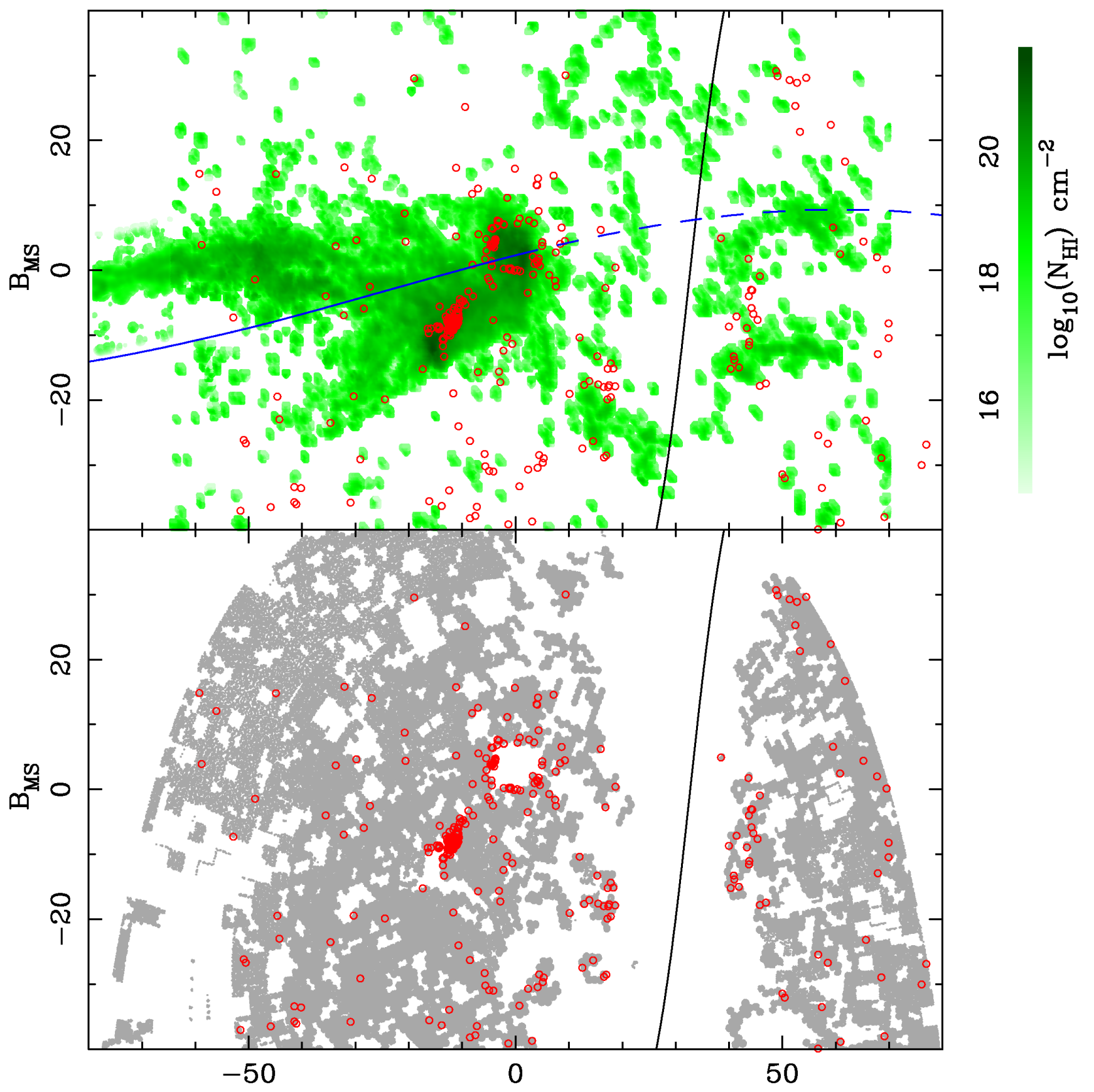

\section{$\Lambda_{\mathrm{MS}}$}

FIG. 15. - Spatial distribution in Magellanic Stream coordinates of our OB candidates (red). The top panel shows the H I column density distribution from Nidever et al. (2010) (green) while the bottom shows the area coverage of our study (grey). The Galactic plane is shown with a black line. The orbit of the LMC is shown with a dark blue line, backward in time (continuous) and forward in time (dashed).

reference is given by the gnomonic projection of celestial coordinates at the location of the target star. The metric distance is defined as $\left(\xi^{2}+\eta^{2}+(\Delta \operatorname{mag} \times 10.0)^{2}\right)^{1 / 2}$. Here, $\xi$ and $\eta$ are the spatial coordinates in degrees, $\Delta m a g$ is the V-magnitude difference between target and reference star, and the factor 10.0 is used to scale magnitude units and spatial units. For each target star, reference stars are sorted in increasing order of the defined distance, and we keep either 50 or 200 reference stars, to represent two different "sizes" of the local reference system. The second local solution uses a strict spatial distance, and keeps the closest 50 or 200 reference objects that also have a $\Delta m a g \leq 1.0 \mathrm{mag}$. The third local solution uses a strict spatial distance; however reference stars are also required to have $\Delta$ mag $\leq 1.0 \mathrm{mag}$ and $\Delta(J-K) \leq 0.3 \mathrm{mag}$. The color restriction aims to minimize color-dependent systematics. The colormagnitude diagram of captured-SMC/targets stars and LMC/reference stars shown by O11, their Fig. 6, indicates that the LMC sample contains some bluer stars 
that are not present in the captured-SMC sample; thus a color-related analysis is a useful check.

With this procedure, we obtain on average (all solu-

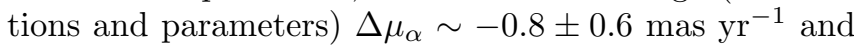
$\Delta \mu_{\delta} \sim-0.2 \pm 0.6$ mas $\mathrm{yr}^{-1}$. Unsatisfied with the rather large uncertainties, we decided to include more information to this determination. We use the formal propermotion errors from the SPM4 catalog for target stars, and thus determine an error-weighted average. This procedure however is prone to any residual proper-motion systematics and, thus, needs to be checked. To do so, we use the LMC sample as a test sample. We randomly choose 285 stars (mimicking the captured-SMC stars) and treat it as a control sample. Since the LMC sample contains 4138 stars, one can devise 14 independent such control samples. The final result for a default control sample is the average of these 14 independent control samples. We apply the same procedures described above, and in principle one should obtain proper-motion differences equal to zero. This is readily obtained when we use simple averages for target control stars; however, when we use an error-weighted average, we obtain a non-zero value in $\Delta \mu_{\delta}$. We believe this is due to unaccounted for systematics in the SPM4. Finally, we use the propermotion differences as obtained by the overall control sample as a zero point offset to be applied to the results obtained with the captured-SMC sample. All these results are summarized in Table 2, where we list the type of solution, the proper-motion difference for the capturedSMC sample and the difference for the control sample which is subtracted from the previous number as a zeropoint correction. Uncertainties are shown in parentheses. The outcome of the zero-point correction and its formal uncertainty are also given. In Figure 17, we plot all these zero-point corrected determinations and their formal uncertainties; they represent the proper-motion difference between the captured-SMC sample and the bona fide LMC sample, at the location of each capturedSMC star. As our final value we take a simple average of all these 24 determinations, and as uncertainty the average uncertainty as given by the weighted-average solutions. This number is $\Delta \mu_{\alpha}=-0.51 \pm 0.30{\text { mas } \mathrm{yr}^{-1}}^{-1}$ and $\Delta \mu_{\delta}=-0.03 \pm 0.29$ mas $\mathrm{yr}^{-1}$; it is represented with a red symbol in Fig. 17. Thus, we obtain a propermotion difference consistent with zero along declination and $1.7 \sigma$ different from zero along right ascension, which, incidentally, is oriented in the direction of the SMC.

\subsection{Kinematical Implications}

Having evidence that the two samples are kinematically distinct, we proceed to analyze the kinematics in a physically meaningful picture. From radial velocities alone and assuming circular motions, O11 proposed two kinematical solutions for the captured-SMC stars: one is a nearly coplanar counter-rotating disk, the other a prograde disk inclined $54^{\circ} \pm 2^{\circ}$ from the LMC disk. Here, we test which of these is preferred given the proper-motion information.

We remind the reader that what we have measured are proper-motion differences between captured-SMC stars and LMC disk stars, locally, i.e., at different regions in the disk which itself rotates. In addition to the rotation of the LMC disk, the bulk space motion of the LMC will be projected onto the proper motions in variable amounts across the disk of the LMC. This is also known as the perspective viewing effect on the proper motions. Other effects include the precession and nutation of the LMC disk; however the amount of precession for instance is rather uncertain, with some studies considering it significant (e.g., vdM02), while others negligible (e.g., K06). The equations for the velocity field across the LMC are given in vdM02, and we use these in a simplified version, i.e., in a coordinate system parallel and perpendicular to the line of nodes. Here, we will neglect the effect of precession/nutation. Also, the space motion of the LMC contributes to the proper motion perpendicular to the line of nodes as a constant (i.e., does not depend on the distance from the center of mass). However, the space motion of the LMC has an effect on the proper motion along the line of nodes that slowly varies with the distance from the center of mass. From Fig. 16, it is apparent that the distribution of captured-SMC stars has two concentrations, one in the north-west and the other in the south-east, roughly aligned with the line of nodes. For all these reasons, we define a new coordinate system, centered on the LMC's center, and rotated from the equatorial system by $52^{\circ}$, i.e., with $\xi$ coordinate along the line of nodes, and positive toward south-east, and $\eta$ coordinate perpendicular to it, and positive toward north-east. We rotate the proper-motion differences to this system, having now a component parallel to the line of nodes $\mu_{\|}$, and another perpendicular to it $\mu_{\perp}$. In this system, we subtract the contribution of the LMC center of mass proper motion, such that the proper motion at each location reflects only the disk rotation and the perspective viewing; note that perspective viewing does not affect the run of $\mu_{\perp}$ with $\xi$. The LMC disk rotates clockwise, with the near side in the north-east. Along the line of nodes, at negative $\xi$, the rotation is projected onto $\mu_{\perp}$ with negative values; the converse is true for positive $\xi$.

We restrict the captured-SMC sample to stars within $|\eta| \leq 3^{\circ}$ (i.e., near the line of nodes), and $1^{\circ}<|\xi| \leq 5^{\circ}$ (i.e., eliminate the few sparse objects in the periphery of the LMC, and a few objects near the center). We also discard stars with proper-motion differences $\left|\Delta \mu_{\|, \perp}\right|>$ 30.0 mas $\mathrm{yr}^{-1}$. In principle, our method is applicable, if all stars are distributed along the line of nodes; however we have a box aligned with the line of nodes, of thickness $6^{\circ}$ and length $10^{\circ}$. Given that the orientation of the line of nodes ranges by about $20^{\circ}$ from various studies (e.g., it is $142^{\circ}$ in $\mathrm{O} 11$, and $122^{\circ}$ in vdM02), our box of given thickness along the line of nodes can be thought of as absorbing this range. The objects are distributed roughly equally at positive and negative $\eta$ coordinate. Therefore, we believe that our approximation works well in the context of our proper-motion errors. These cuts restrict our sample to between $\sim 200$ and 210 capturedSMC stars for the various solutions shown in Table 2.

To calculate the proper motions in this new system, we need a model for the rotation of the LMC disk, as well as the tangential and line-of-sight velocity of the LMC's center of mass, and the angle between the line of nodes and the direction of the tangential velocity. For the rotation, we adopt the model described in O11, in which the disk has an inclination angle of $34.7^{\circ}$ (van der Marel \& Cioni 2001), a rotation curve that increases 
linearly from 0 to $87 \mathrm{~km} \mathrm{~s}^{-1}$ between 0 and $2.4 \mathrm{kpc}$ from the LMC's center and is flat afterward. The position angle of the line of nodes is $\theta=142^{\circ}$ (measured from north to east). We assume that the LMC's center is at $(R A, D e c)=.\left(81.90^{\circ},-69.87^{\circ}\right)$ and the distance to the LMC is $50.1 \mathrm{kpc}(\mathrm{vdM} 02)$. The tangential velocity of the center of mass and its orientation is that from V10, and its line of sight velocity is $262.1 \pm 3.4 \mathrm{~km} \mathrm{~s}^{-1}$ (vdM02).

In Figure 18, we show the proper-motions with respect to the LMC's center of mass $\mu_{\perp}$ (top) and $\mu_{\|}$(bottom) of the captured-SMC stars as a function of $\xi$ coordinate. These are from the local solution \#1 with 50 reference stars (see Tab. 2), i.e., one treatment of the six. The shaded areas represent the 1-sigma error of linear fits to the data. We also show the rotation curve of the LMC disk (red line), the rotation curve of a counter-rotating disk (dashed red line) (i.e., in the same plane as the LMC disk, and with the same amplitude rotation, but in the opposite sense), and that of a prograde disk with zero inclination angle, and amplitude of $200 \mathrm{~km} \mathrm{~s}^{-1}$ (blue line). In the top plot, these models are easily distinguishable, however, in the bottom plot they depart slightly from zero, and nearly coincide, indicating that rotation has a very small contribution to $\mu_{\|}$.

In Figure 19 we show a zoomed-in version of Fig. 18 , where the data points are replaced with the errorweighted average values of the proper motions at positive and negative $\xi$. Each weighted average value is from one of the six different local-type solutions (Tab. 2). The $\pm 1 \sigma$ range of the $\xi$ coordinate at positive and negative $\xi$ is shown with grey lines. The representation of the three model disks is the same as in Fig. 18.

The two solutions proposed by O11 for the capturedSMC stars are as follows. The first is a disk of similar inclination to that of the LMC, specifically $20^{\circ} \pm 3^{\circ}$, but rotating in the opposite sense, with the line of nodes oriented at $\theta=177^{\circ} \pm 7^{\circ}$. They refer to this solution as the counter-rotating disk. Although our model for the counter-rotating disk differs slightly from the O11 one, namely by $\sim 15^{\circ}$ in inclination, and $\sim 35^{\circ}$ in the orientation of the line of nodes, these slight changes do not affect our conclusions. For instance, the rotation velocity is projected onto $\mu_{\perp}$ as $V_{\text {rot }} \times \cos i$, where $i$ is the inclination angle. A change of $i=34.7^{\circ}$ to $20^{\circ}$ implies a change of $\sim 10 \%$ in $V_{\text {rot }} \times \cos i$, or of the order of 0.04 mas $\mathrm{yr}^{-1}$. A change of $35^{\circ}$ in the orientation of the line of nodes, implies a change of $18 \%$ in $V_{\text {rot }} \times \cos i$, or 0.05 mas $\mathrm{yr}^{-1}$. These values are well below our uncertainties.

From Fig. 19, top panel, it is clear that the counterrotating disk model is disfavored by the observations, in fact it is discrepant at a $4.9 \sigma$ level. The second solution proposed by $\mathrm{O} 11$, is a disk inclined from the plane of the sky at $-19^{\circ} \pm 2^{\circ}$, with a similar orientation of the line of nodes as in their first solution, and rotating in the same sense as the LMC disk. Our proper-motions support this solution, as the sense of rotation is the correct one: positive slope in the run of $\mu_{\perp}$ with $\xi$. The proper-motion amplitude should be larger than for prograde rotation with $i=34.7^{\circ}$, since the inclination angle is smaller in absolute value, but only by $\sim 0.04$ mas $\mathrm{yr}^{-1}$ (see above). The limiting case of a disk with $i=0^{\circ}$ and amplitude $V_{\text {rot }}=200 \mathrm{~km} \mathrm{~s}^{-1}$ is shown with a blue line. The proper motions imply prograde rotation in excess of

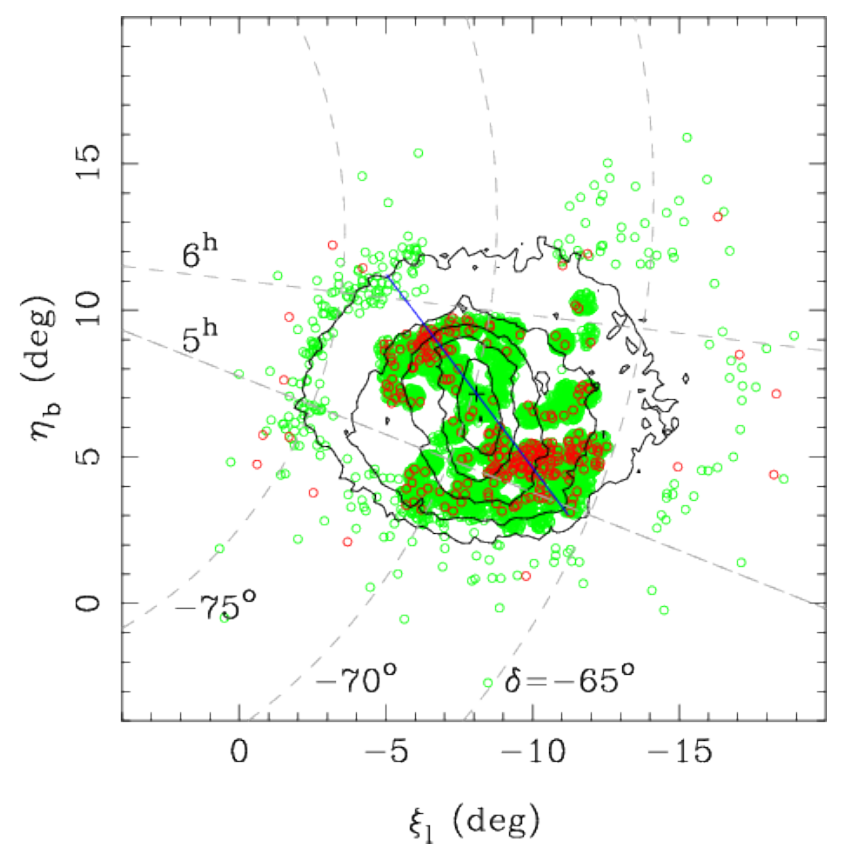

FIG. 16. - Spatial distribution of the captured-SMC sample from O11 (red), and bona fide LMC sample (green). M-giant density contours are shown in black. The coordinate system is the same as in Fig. 8-10. The LMC's center is marked with a cross. The line of nodes of the LMC disk is shown with a blue line.

$200 \mathrm{~km} \mathrm{~s}^{-1}$. However, it is very likely that the motions of captured-SMC stars are non-circular. That this may be the case, is also implied by the run of $\mu_{\|}$with $\xi$ (Fig. 19 , bottom). The observations disagree with all models at a $\sim 2 \sigma$ level.

The escape velocity from the LMC at a distance of 2.5 kpc from its center $\left(\xi \sim 2.9^{\circ}\right.$, from Fig. 19) is $V_{\text {esc }}=$ $148 \mathrm{~km} \mathrm{~s}^{-1}$. This is estimated by adopting a Plummer potential for the LMC with a total mass of $10^{10} \mathrm{M}_{\odot}$, and a scale radius of $3 \mathrm{kpc}(\mathrm{DB} 12)$. This is a rather low mass for the LMC; a factor of two larger mass would provide an escape velocity of $210 \mathrm{~km} \mathrm{~s}^{-1}$. Our proper motions thus indicate that the captured-SMC stars are near the upper limit of velocities for a bound status.

\section{DISCUSSION}

\subsection{Implications of the Spatial Distribution of $O B$ Candidates}

Our sample of OB candidates was purposely built with rather restrictive constraints: we aimed to produce a reliable map of the spatial distribution over an expansive area, rather than a complete sample of candidates. For instance, the comparison with 6X 2MASS indicates that our errors in $J$ magnitudes, as given by 2MASS at the faint end, tend to overlook candidates rather than contaminate our sample with non-candidates. The color cuts also were designed to secure the bluest, i.e., youngest objects in our sample, and to eliminate foreground subdwarfs which are more frequent at B-type than O-type (Vennes et al. 2011 and references therein). These types of stars have been studied starting in the early nineties, with the photographic work by Irwin et al. (1990) in two UK Schmidt fields located between the Clouds. Deep, follow-up CCD photometry on small subfields selected from the Irwin et al. (1990) study by Demers \& Bat- 
TABLE 2

Proper-MOTION RESUlts

\begin{tabular}{|c|c|c|c|c|}
\hline Solution & \multicolumn{2}{|l|}{$\Delta \mu_{\alpha}$} & \multicolumn{2}{|l|}{$\Delta \mu_{\delta}$} \\
\hline $\begin{array}{r}200, \text { box } 20 \\
50, \text { box } 20 \\
200, \text { box } 40 \\
50, \text { box } 40\end{array}$ & $\begin{array}{l}-0.85(0.53)-[-0.20(0.11) \\
-0.43(0.54)-[-0.13(0.11) \\
-1.37(0.70)-[-0.20(0.19) \\
-1.12(0.70)-[-0.09(0.19)\end{array}$ & $\begin{aligned} & \text { local \#1, ave } \\
= & -0.65 \pm 0.54 \\
= & -0.30 \pm 0.55 \\
= & -1.17 \pm 0.72 \\
= & -1.03 \pm 0.70\end{aligned}$ & $\begin{array}{l}-0.01(0.50)-[-0.06(0.09)] \\
-0.10(0.50)-[-0.07(0.09)] \\
-0.64(0.69)-[-0.21(0.09)] \\
-0.69(0.69)-[-0.28(0.11)]\end{array}$ & $\begin{array}{l}=+0.04 \pm 0.51 \\
=-0.03 \pm 0.51 \\
=-0.43 \pm 0.69 \\
=-0.41 \pm 0.70\end{array}$ \\
\hline $\begin{array}{r}200, \text { box } 20 \\
50, \text { box } 20 \\
200, \text { box } 40 \\
50, \text { box } 40\end{array}$ & $\begin{array}{l}0.87(0.27)-[-0.08(0.13)] \\
-0.35(0.29)-[+0.01(0.13)] \\
-0.58(0.25)-[-0.02(0.16) \\
-0.24(0.27)-[+0.08(0.15)\end{array}$ & $\begin{aligned} & \text { local \#1, w.a } \\
= & -0.79 \pm 0.30 \\
= & -0.36 \pm 0.32 \\
= & -0.56 \pm 0.30 \\
= & -0.32 \pm 0.31\end{aligned}$ & $\begin{array}{l}+0.60(0.27)-[+0.55(0.11)] \\
+0.53(0.28)-[+0.44(0.11)] \\
-0.07(0.25)-[+0.46(0.09)] \\
-0.03(0.27)-[+0.32(0.11)]\end{array}$ & $\begin{array}{l}=+0.05 \pm 0.29 \\
=+0.09 \pm 0.30 \\
=-0.53 \pm 0.26 \\
=-0.35 \pm 0.29\end{array}$ \\
\hline $\begin{array}{r}200, \text { box } 20 \\
50, \text { box } 20 \\
200, \text { box } 40 \\
50, \text { box } 40\end{array}$ & $\begin{array}{l}-0.45(0.53)-[-0.15(0.11) \\
-0.18(0.53)-[-0.21(0.10) \\
-1.21(0.70)-[-0.02(0.18) \\
-1.02(0.70)-[-0.16(0.19)\end{array}$ & $\begin{aligned} & \text { local2, ave. } \\
= & -0.30 \pm 0.54 \\
= & +0.03 \pm 0.54 \\
= & -1.19 \pm 0.72 \\
= & -0.86 \pm 0.72\end{aligned}$ & $\begin{array}{l}+0.14(0.49)-[-0.13(0.10)] \\
-0.03(0.50)-[-0.17(0.10)] \\
-0.22(0.66)-[-0.25(0.09)] \\
-0.75(0.70)-[-0.28(0.11)]\end{array}$ & $\begin{array}{l}=+0.27 \pm 0.50 \\
=+0.14 \pm 0.51 \\
=+0.03 \pm 0.67 \\
=-0.47 \pm 0.71\end{array}$ \\
\hline $\begin{array}{r}200, \text { box } 20 \\
50, \text { box } 20 \\
200, \text { box } 40 \\
50, \text { box } 40\end{array}$ & $\begin{array}{l}-0.42(0.27)-[-0.02(0.12)] \\
-0.22(0.28)-[+0.01(0.12) \\
-0.24(0.26)-[+0.16(0.15) \\
-0.07(0.27)-[+0.12(0.15)]\end{array}$ & $\begin{aligned} & \text { local2, w.a. } \\
= & =-0.40 \pm 0.29 \\
= & -0.23 \pm 0.30 \\
= & -0.40 \pm 0.30 \\
= & -0.19 \pm 0.31\end{aligned}$ & $\begin{array}{l}+0.62(0.27)-[+0.34(0.10)] \\
+0.36(0.29)-[+0.16(0.09)] \\
+0.16(0.26)-[+0.28(0.11)] \\
-0.05(0.27)-[+0.09(0.10)]\end{array}$ & $\begin{array}{l}=+0.28 \pm 0.29 \\
=+0.20 \pm 0.30 \\
=-0.12 \pm 0.28 \\
=-0.14 \pm 0.29\end{array}$ \\
\hline $\begin{array}{r}200, \text { box } 20 \\
50, \text { box } 20 \\
200, \text { box } 40 \\
50, \text { box } 40\end{array}$ & $\begin{array}{l}-0.45(0.54)-[-0.15(0.08) \\
-0.06(0.51)-[-0.16(0.10) \\
-1.15(0.72)-[-0.10(0.17) \\
-1.13(0.70)-[-0.12(0.19)\end{array}$ & $\begin{aligned} & \text { local3, ave. } \\
= & -0.30 \pm 0.54 \\
= & +0.10 \pm 0.52 \\
= & -1.05 \pm 0.74 \\
= & -1.01 \pm 0.72\end{aligned}$ & $\begin{array}{l}+0.21(0.50)-[-0.01(0.10)] \\
+0.32(0.50)-[-0.08(0.10)] \\
-0.31(0.67)-[-0.11(0.11)] \\
-0.26(0.69)-[-0.25(0.11)]\end{array}$ & $\begin{array}{l}=+0.22 \pm 0.51 \\
=+0.40 \pm 0.51 \\
=-0.20 \pm 0.68 \\
=-0.01 \pm 0.70\end{array}$ \\
\hline $\begin{array}{r}200, \text { box } 20 \\
50, \text { box } 20 \\
200, \text { box } 40 \\
50, \text { box } 40\end{array}$ & $\begin{array}{l}-0.48(0.28)-[-0.01(0.12) \\
+0.00(0.29)-[+0.02(0.13) \\
-0.29(0.26)-[+0.15(0.16) \\
-0.09(0.27)-[+0.15(0.15)\end{array}$ & $\begin{aligned} & \text { local3, w.a. } \\
= & -0.47 \pm 0.30 \\
= & -0.02 \pm 0.32 \\
= & -0.44 \pm 0.30 \\
= & -0.24 \pm 0.31\end{aligned}$ & $\begin{array}{l}+0.55(0.28)-[+0.39(0.11)] \\
+0.52(0.29)-[+0.22(0.09)] \\
+0.09(0.26)-[+0.34(0.11)] \\
+0.14(0.27)-[+0.12(0.10)]\end{array}$ & $\begin{array}{l}=+0.16 \pm 0.30 \\
=+0.30 \pm 0.30 \\
=-0.25 \pm 0.28 \\
=+0.02 \pm 0.29\end{array}$ \\
\hline
\end{tabular}

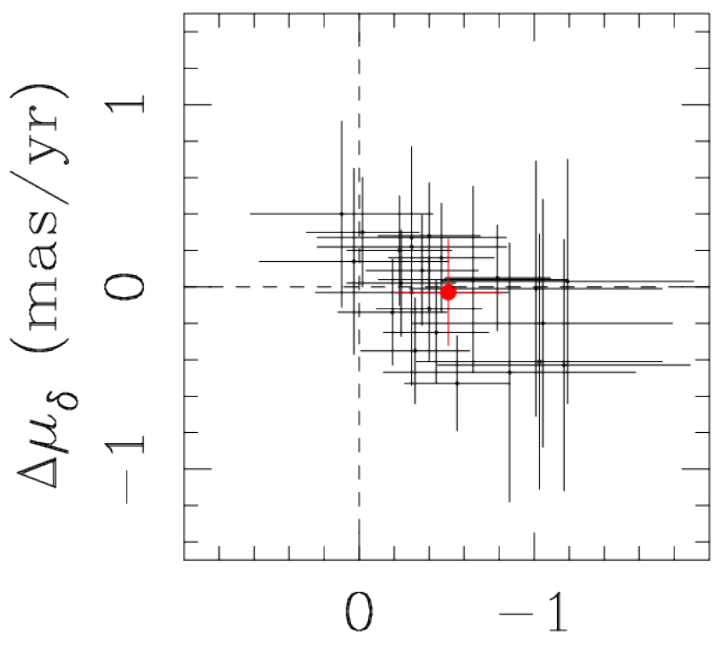

$\Delta \mu_{\alpha}(\operatorname{mas} / \mathrm{yr})$

FIG. 17. - Twenty-four determinations of the proper-motion difference between the captured-SMC sample and the LMC sample; the average value of these determinations is shown in red.

tinelli (1998) indicated the existence of associations with ages as young as 10-25 Myr. Our work supports this notion, although ages as old as $\sim 90$ Myr are allowed by our color cuts (main-sequence lifetime of a B5 type star). The notion that very young stars $(\sim 10-30$ Myr $)$ are present in the eastern part of the SMC wing is supported by the finding of three high mass X-ray binaries with optical counterparts of confirmed (Kahabka \& Hilker 2005) and implied (McBride et al. 2010) Be spectral type. A 10 Myr-lived star located some $7 \mathrm{kpc}\left(6.4^{\circ}\right)$ from the SMC's center requires an excess velocity of $700 \mathrm{~km} \mathrm{~s}^{-1}$ to travel this distance. Kahabka \& Hilker (2005) measure the radial velocity of their Be optical counterpart to be no different than $30 \mathrm{~km} \mathrm{~s}^{-1}$ from the SMC's velocity. Therefore, these young stars must have formed in situ rather than forming in the inner regions of the SMC, and migrating to their current location.

Most remarkably, the LMC periphery shows two stellar concentrations at the ends of the bar, and a well populated distribution in the north-east region. In some nearby barred spiral galaxies (e.g., Sheth et al. 2002), star-formation activity appears enhanced at the ends of the bar, indicating perhaps the beginning of rings. Our Milky Way too has been shown to have young and massive (ages $\leq 50 \mathrm{Myr}$ and masses $\geq 10^{4} \mathrm{M}_{\odot}$ ) stellar clusters at the near and far ends of the bar (Davies et al. 2012 and references therein). In the case of the LMC however, we have a clear case of a recent interaction with the SMC, which is responsible for the newly formed stars in the LMC's periphery, as well as captured-SMC stars in the LMC. We also know that the recent star-formation history of the Clouds is coupled, noting the recent study of the ages of young $(<1 \mathrm{Gyr})$ clusters in the Clouds by Glatt et al. (2010). Their study indicates that there were 

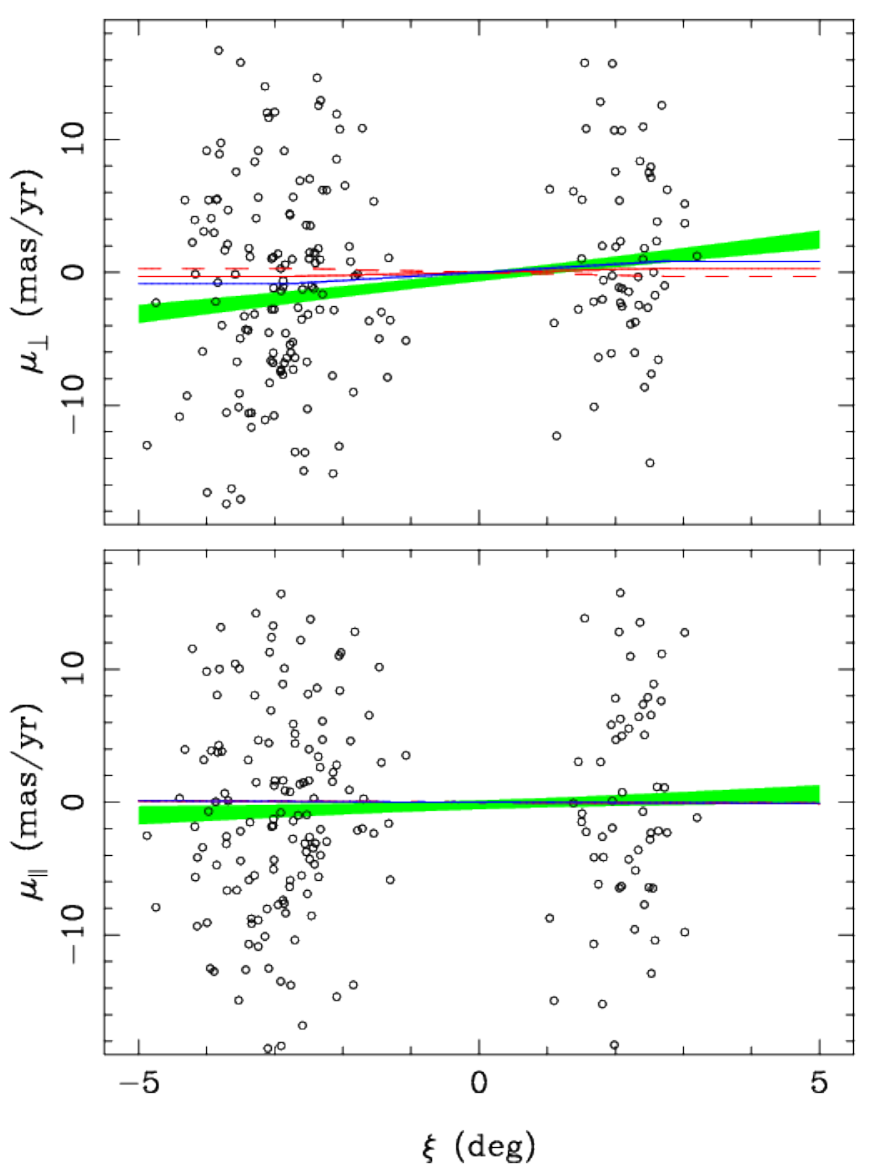

Fig. 18. - Proper motions perpendicular (top) and parallel (bottom) to the line of nodes, as a function of position along the line of nodes (open symbols). Proper motions are with respect to the LMC's center of mass proper motion. The green shaded areas show the $1 \sigma$-error of a linear fit to the data. The rotation of the LMC disk is shown with a red line; that of a counter-rotating disk with a dashed red line. A positive slope in $\mu_{\perp}$ indicates prograde rotation. The blue line indicates prograde rotation with zero inclination angle, and amplitude $V_{\text {rot }}=200 \mathrm{~km} \mathrm{~s}^{-1}$. In the bottom plot, these three models nearly coincide. The data points represent one of the six types of solutions presented in Tab. 2 .

two periods of enhanced star formation in both Clouds, and that these are nearly coincident: in the LMC at 125 Myr and $800 \mathrm{Myr}$, and in the SMC at $160 \mathrm{Myr}$ and 630 Myr. Clearly, the recent epoch of enhanced star formation in both Clouds is due to the recent interaction implied by the orbits.

For the first time, we present the prospect of finding newly formed stars in the LA. The existence of two OBcandidate clumps in regions of high $\mathrm{H}$ I column density in the LA needs to be further explored spectroscopically for confirmation. The LA is the most challenging structure to reproduce in models of the Magellanic system, and as discussed by previous studies (e.g., DB12), the evolution of this material in the recent past (300 Myr) should be influenced by hydrodynamical interactions with the MW hot gaseous halo (i.e., ram pressure).

\subsection{Kinematical Evidence Regarding the Most Recent Interaction of the Clouds}

In order to explore their recent interaction, we use the proper motions of the Clouds as measured by K06 and V10 to integrate their orbits back in time as individual

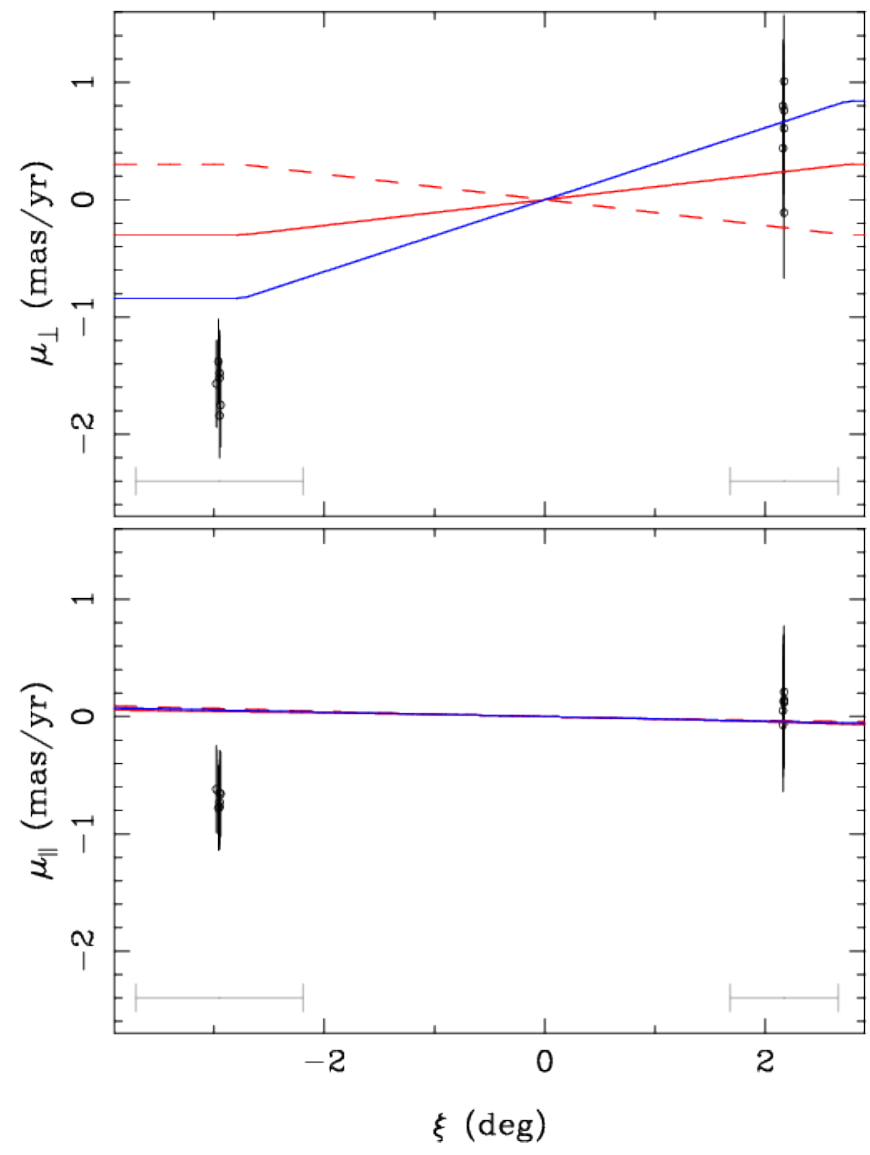

FIG. 19. - The data points represent weighted averages for the six types of local solutions (see Tab. 2 and text) at positive and negative $\xi$. The rotation curve of the LMC disk is shown in red, that of a counter-rotating disk in dashed red, and that of a prograde disk with zero inclination and amplitude $V_{\text {rot }}=200 \mathrm{~km} \mathrm{~s}^{-1}$ in blue. The $\pm 1 \sigma \xi$ range of the dta is also shown with grey lines.

point masses in a realistic, three-component Milky-Way gravitational potential (Johnston et al. 1995). We then calculate the minimum separation between the Clouds (impact parameter), the time as well as the relative velocity at minimum separation. We also calculate the angle between the relative velocity and the LMC disk at minimum separation. We assume the LMC's disk has an inclination of $34.7^{\circ}$ and the position angle of the line of nodes is $\theta=142^{\circ}$ (e.g., O11). The present disk inclination corresponds to an orientation in Galactocentric coordinates that is assumed not to change with time. As we are interested in the most recent interaction between the Clouds, we integrate back in time for only $400 \mathrm{Myr}$, compared to several Gyr-period orbits (or unbound orbits, Besla et al. 2007).

We vary the input proper motions according to their formal errors, (where for V10 we use the errors as obtained in the Clouds' relative proper-motion estimation, i.e., 0.11 mas $\mathrm{yr}^{-1}$ per Cloud). Distances, radial velocities and the Clouds' locations are taken from V10. Proper-motion errors are assumed to have a Gaussian distribution; the distances and radial velocities of the Clouds are assumed to have zero errors. We integrate 1000 such realizations, and determine the range for the inner $68.2 \%$ of the values. Results are summarized in Table 3, where the first column indicates the reference 
to the proper-motion measurement, the second column shows the minimum separation and the third shows the time when the encounter occured. The fourth column shows the relative velocity at minimim separation, and the fifth column shows the angle between the relative velocity and the LMC disk, at minimum separation. We

TABLE 3

Parameters of the Most Recent Interaction

\begin{tabular}{rrrrr}
\hline & $\mathrm{d}_{\min }(\mathrm{kpc})$ & $\mathrm{t}(\mathrm{Myr})$ & $\mathrm{V}_{\text {rel }} \mathrm{km} \mathrm{s}^{-1}$ & Angle $(\mathrm{deg})$ \\
\hline V10 & $11.6_{-2.1}^{+6.2}$ & $223_{-108}^{+35}$ & $91.6_{-22.3}^{+54.6}$ & $125.3_{-10.3}^{+4.7}$ \\
$\mathrm{~K} 06$ & $13.9_{-4.7}^{+8.9}$ & $234_{-176}^{+20}$ & $82.4_{-15.4}^{+80.8}$ & $110.5_{-35.5}^{+10.5}$ \\
\hline
\end{tabular}

note that in the K06 tests, 47 realizations place the minimum separation in the future, i.e., the Clouds are currently approaching one another. Also, the distribution of the minimum separation is rather flat between 9 and $20 \mathrm{kpc}$, and increases toward larger minimum separations. In the V10 tests, there are only 8 such occurrences, and the distribution of the minimum separation has a peak at $\sim 12 \mathrm{kpc}$; overall the V10 parameters are better constrained than those using K06. This simple approach neglects dynamical friction of the Clouds with the Milky Way and the gravitational interaction between the Clouds. Since the tidal radius of the LMC is $\sim 15$ $\mathrm{kpc}$, the gravitational effect of the LMC on the SMC will become important for separations smaller than this value. As such, our estimates of the minimum separation are to be regarded as upper limits. Our simple approach indicates that the impact angle of the SMC with respect to the LMC disk is $35^{\circ}{ }_{-10}^{+5}$ away from being coplanar according to the V10 proper motions, and smaller assuming K06 (Table 3). That the impact is not coplanar is also implied by our analysis of the O11 captured-SMC stars, which favors the interpretation that thses to move in a plane inclined by $\sim 54^{\circ}$ from LMC's disk.

To fully understand this interaction, N-body studies of both Clouds in a realistic MW potential are needed. One such recent study is that of DB12, where the interaction is modeled after a preliminary thorough search in the orbital parameter space that was centered on the V10 proper motions. Their search for a realistic model is further constrained by key observational facts such as the recent $(\leq 2 \mathrm{Gyr})$ formation of the binary pair necessary for the formation of the MS, the actual spatial distribution and kinematics of the MS, and the current positions and velocities of the Clouds. The only drawback of this major study that aims to reproduce the MS, Bridge, and SMC morphology, is that the LMC's morphology was not captured, nor was it meant to be, in accordance with the initial goals of the study; that is the SMC is described by an N-body system, while the LMC and MW have analytic potentials. The best model of DB12 places the most recent interaction at a minimum separation of 6.6 kpc some $t=260 \mathrm{Myr}$ ago. We also mention the recent work by Besla et al. (2012) who use an N-body smoothed-particle hydrodynamics (SPH) code to model the interaction, where both Clouds are modeled as $\mathrm{N}$ body/SPH systems with disks, and the MW has a static potential. Besla et al. (2012) focus on describing the particular morphology of the LMC in light of a collision between the Clouds that took place in the recent past.
The LMC model has a bar before the interactions take place. However, their modeling does not reproduce the current position and velocity of the SMC. The model is constrained by the orbit of the LMC as measured by K06, and other facts that point to a recent interaction between the Clouds. Among these are: the coupled recent star-formation history of the Clouds (e.g., Harris \& Zaritsky 2009), the off-center stellar bar in the LMC (the center of the bar is offset by $\sim 1.2^{\circ}$ from the kinematic center of $\mathrm{H} \mathrm{I}$; vdM02), the Bridge and its gaseous and stellar makeup, and the existence of captured-SMC stars in the LMC (O11). Their description of the interaction accounts for many features of the particular morphology of the LMC, with the most recent interaction having occured 100 Myr ago and being a central collision at high inclination with respect to the LMC's disk.

The questions that remain to be answered are related to the particulars of this interaction, such as impact parameter and impact angle with respect to the LMC's disk. These will determine many of the properties of the present day LMC, SMC and Bridge. We qualitatively explore the N-body/SPH models by Berentzen et al. (2003) that describe the interaction between a barred disk galaxy and a small (20\% mass of the disk galaxy) spherical perturber. The gas amounts to $30 \%$ of the disk mass. These models analyze nearly perpendicular passages with respect to the disk plane, and three impact parameters: central $(0 \mathrm{kpc})$, minor-axis impact $(3 \mathrm{kpc})$ and major-axis impact $(6 \mathrm{kpc})$. All these interactions produce expanding ring structures, off-center bars, spokes and other asymmetries in the stars and gas, and are unlike coplanar interactions that primarily influence the strength of the existing bar of the host galaxy (Berentzen et al. 2004). Among the three classes of models presented by Berentzen et al. (2003), the central and minor-axis impact have their stellar bar destroyed in $\sim 200$ Myr. This is not the case of the LMC, which has a strong bar seen in its M-giant population. In the major-axis impact, the stellar bar survives, has its maximum displacement $(2.4 \mathrm{kpc})$ from the initial position $100 \mathrm{Myr}$ after impact, and recenters after $600 \mathrm{Myr}$ (some two disc rotations). The impact produces a radially expanding density wave which becomes first visible in the gas. Since the impact is off-center, the symmetry of the gaseous ring is broken when it encounters the stellar bar or spiral arms. Nevertheless, gaseous density enhancements in ring-like shapes and at the ends of the bar appear to form (see Fig. 17 and 22 in Berentzen et al. 2003, see also simulations by Bekki \& Chiba 2007, their Figs 4 and 5). Thus, the distribution of the OB candidates in the LMC's periphery, the timing of the interaction, and the characteristics of the bar point to an off-center collision of moderate to high inclination with respect to the host's disk. Although the $1 \sigma$ lower limit of the impact parameter is $\sim 9 \mathrm{kpc}$ from our simple analysis of the proper-motion data, this may not represent a problem when more realistic models are considered (e.g., DB12).

The recent interaction of the Clouds also tidally strips material from the SMC disk in the form of gas and stars and forms the observed Magellanic Bridge. This is clearly seen in the recent work of Besla et al. (2012) and DB12, as well as in some earlier work. For the reasons mentioned above, we follow in more detail the prediction of the DB12 work. They predict that the interaction at 
$\sim 200$ Myr ago produced two tidal structures, one filling the region between the Clouds, and representing the observed Bridge, and the other - the Counter Bridge - extending away from the SMC, but rather aligned with the line of sight to the SMC, and thus hidden behind the SMC. The simulations show that the Counter Bridge has an extent of $\sim 20 \mathrm{kpc}$. It is known that the SMC has a significant depth along the line of sight $12-14$ kpc (Subramanian \& Subramanian 2012, Crowl et al. 2001). Here we suggest the possibility that the two branches of $\mathrm{OB}$ candidates extending from the western part of the SMC wing and surrounding the Cloud are part of this Counter-Bridge structure. Spectroscopic information to secure distances and radial velocities is necessary to further explore this matter. The DB12 model also predicts the existence of tidally stripped stars in the Bridge. We note that the photometric study by Harris (2007) that sampled twelve fields in the Bridge, found no older stars. However, his fields were placed along the ridge line of the $\mathrm{H}$ I density, which appears to be offset from the OB candidates. One field, (\#14, (RA, Dec.) $\left.=\left(03^{h} 18^{m},-73^{\circ} 18^{\prime}\right)\right)$ from his study was placed $\sim 1^{\circ}$ north from the $\mathrm{H}$ I ridge line, i.e., closer to the location of our OB candidates. Monelli et al. (2011) reinvestigate two of the Harris (2007) fields, including field \#14 with deep and wide-field photometry. Their Fig. 3 shows a well-defined main sequence in field \#14 as well as in field \#13, thus reinforcing the tidal nature of the Bridge.

\section{SUMMARY}

Based on photometry from GALEX, 2MASS, SPM4 and APASS and on proper motions from SPM4, we select a sample of distant (low proper-motion) OB-type candidates in a 7900 square degree area, for the purpose of mapping recently formed stars in the Magellanic Clouds' periphery and Bridge, and to explore the presence of such stars in the Leading Arm and Magellanic Stream. We find a well populated LMC periphery with two prominent clumps at the ends of the LMC bar, and the northern side being more abundant than the south. In agreement with earlier work, we also find a rich SMC wing that continues eastward in a narrow band toward the LMC to RA $\sim 3.4^{h}$. This path is offset by $1^{\circ}-2^{\circ}$ from the $\mathrm{H}$ I ridge line in the Bridge. A group of 2-3 candidates is present in the LMC periphery at R.A. $=4.2^{h}$ in the direction of the band extending from the SMC wing, and suggesting that this is the site where material from the SMC is funneled into the LMC. The western part of the SMC wing splits into two branches that partly surround the SMC; they may be part of the Counter Bridge tidal structure produced in the most recent interaction between the Clouds as modeled by DB12.

We also find clumps of $\mathrm{OB}$ candidates in the LA and coincident with high $\mathrm{H}$ I density regions. Although these clumps are located at low Galactic latitudes, the known spiral structure of the MW does not offer a compelling alternative explanation. A few isolated OB candidates are also seen in the MS. Spectroscopic investigation of these candidates is most desirable.

Simple calculations based on the Clouds' proper motions suggest that the most recent interaction took place some 200 Myr ago, with an impact parameter between 9 and $18 \mathrm{kpc}$ (from the V10 proper motions). The sophisticated modeling by DB12 indicates a similar impact time, with an impact parameter of $6.6 \mathrm{kpc}$. The orbits of the Clouds, taken together with aspects such as the off-center LMC bar, the captured SMC stars in the LMC (O11), and the configuration of the OB candidates in the LMC, imply that this interaction was an off-center, moderate to high-inclination collision between the SMC and LMC's disk.

We also analyze the SPM4 proper motions of the radial-velocity sample from O11 of $\sim 5900$ red supergiants, oxygen- and carbon-rich asymptotic giant branch stars and other giants. O11 showed that $5 \%$ of these stars have distinct kinematics from the rotation of the LMC disk, and proposed that these stars are captured from the SMC. O11 also show that the metallicities of 30 such stars are compatible with the metallicity of the SMC's outer regions. Under the assumption of circular motions, O11 propose two kinematical solutions for the captured-SMC stars: a counter-rotating disk, i.e., similar plane with that of the LMC disk, and retrograde rotation, and a prograde rotation of a disk some $54^{\circ}$ away from the LMC disk plane, i.e., at $-19^{\circ}$ inclination with the plane of the sky. The proper motions indicate that 1) the captured-SMC group is kinematically distinct from the LMC disk sample, 2) the counter-rotating disk solution proposed by $\mathrm{O} 11$ on radial-velocities alone is rejected at $>4 \sigma$ level, while their second solution of a prograde rotation at inclination $-19^{\circ}$ appears to be a compatible description, although the assumption of circular motions may be unrealistic, and 3) the implied orbital velocities for the captured-SMC star indicate a marginally bound status to the LMC. Our proper-motion analysis thus reinforces the hypothesis put forward by $\mathrm{O} 11$, that these are not bona fide LMC stars, but rather captured from the SMC during their close interaction in the recent past (e.g., DB12 and references therein).

We acknowledge support by the NSF through grant AST04-0908996. We thank Stephane Vennes for making available the model colors for main sequence and white dwarf stars. We are grateful to Knut Olsen for providing the radial-velocity catalog in the LMC, and to Mathew Templeton from AAVSO for making available the APASS-DR3 catalog. We thank David Nidever for making available the $\mathrm{H}$ I column density data and for helpful discussions regarding this study. This publication makes use of data products from the Two Micron All Sky Survey, which is a joint project of the University of Massachusetts and the Infrared Processing and Analysis Center/California Institute of Technology, funded by the NASA and the NSF. This research was made possible through the use of the AAVSO Photometric All-Sky Photometric Survey, funded by Robert Martin Ayers Sciences Fund.

\section{REFERENCES}

Baume, G, Carraro, G. \& Momany, Y. 2009, MNRAS, 398, 221

Berentzen, I., Athanassoula, E., Heller, C. H., \& Fricke, K. J. 2003, MNRAS, 341, 343
Berentzen, I, Athanassoula, E., Heller, C. H., \& Fricke, K. J. 2004,

MNRAS, 347,220 
Besla, G., Kallivayalil, N., Hernquist, L., Roberston, B., Cox, T. J., van der Marel, R. P., \& Alcock, C. 2007, ApJ, 668, 949

Bekki, K. \& Chiba, M. 2007, PASA, 24, 21

Besla, G., Kallivayalil, N., Hernquist, L., Robertson, B, Cox, T, J., van der Marel, R. P., \& Alcock, C. 2007, ApJ, 668, 949

Besla, G., Kallivayalil, N., Hernquist, L., van der Marel, R. P., Cox, T. J., \& Keres, D. 2010, ApJ, 721, L97

Besla, G., Kallivayalil, N. Hernquist, L., van der Marel, R. P., Cox, T. J., Keres, D. 2012, astroph 1201.1299, MNRAS, in press

Bianchi, L., Efremova, B., Herald, J., Girardi, L., Zabot, A., Marigo, P., \& Martin, C. 2011, MNRAS, 441, 2770

Bica, E., Bonatto, C., Dutra, C. M., \& Santos, J. F. C. 2008, MNRAS, 389, 678

Bonanos, A. Z. et al. 2009, AJ, 138, 1003

Bonanos, A. Z. et al. 2010, AJ, 140, 416

Carraro, G., \& Costa, E. 2009, A\&A, 493, 71

Connors, T. W., Kawata, D., \& Gibson, B. K. 2006, MNRAS371, 108

Costa, E., Méndez, R. A., Pedreros, M. H., Moyano, M., Gallart, C., Noel, N., Baume, G., \& Carraro, G. 2009, AJ, 137, 4339

Crowl, H. H., Sarajedini, A., Piatti, A. E., Geisler, D., Bica, E., Claria, J. J., \& Santos, J. F. C. 2001, AJ, 122, 220

Davies, B., de la Fuente, D., Najarro, F., Hinton, J. A., Trombley, C., Figer, D. F., \& Puga, E. 2012, MNRAS, 419, 1860

Demers, S., \& Battinelli, P. 1998, AJ, 115, 154

Diaz, J. D., \& Bekki, K. 2012, in press, (astro-ph 1112.6191)

Gardiner, L. T., \& Hatzidimitriou, D. 1992, MNRAS, 257, 195

Gardiner, L. T. \& Noguchi, M. 1996, MNRAS, 278, 191

Girard, T. M., van Altena, W. F., Zacharias, N., Vieira, K., CasettiDinescu, D. I., Castillo, D., Herrera, D., Lee, Y. S., Beers, T. C., Monet, D. G., \& López, C. E. 2011, AJ, 142, 12

Glatt, K., Grebel, E. K., \& Koch, A. 2010, A\&A, 517, 50

Harris, J., 2007, ApJ, 658, 345

Harris, J. \& Zaritsky, D. 2009, AJ, 138, 1243

Hasche, R., Grebel, E. K., \& Duffau, S. 2011, AJ, 141, 158

Henden, A. A., Levine, S. E., Terrell, D., Smith. T. C., \& Welch, D. L. 2011, AAS, 218, 126.01

Irwin, M. J., Demers, S., \& Kunkel, W. E. 1990, AJ, 99, 191

Johnston, K. V., Spergel, D. N., \& Hernquist, L. 1995, apj, 451, 598

Kahabka, P., \& Hilker, M. 2005, A\&A, 435, 9

Kallivayalil, N., van der Marel, R. P., Alcock, C., Axelrod, T. Cook, K. H., Drake, A. J., \& Geha, M. 2006, ApJ, 638, 772 (K06)
Kallivayalil, N., van der Marel, R. P., \& Alcock, C. 2006, ApJ, 652, 1213 (K06)

Kinman, T. D., Salim, S. \& Clewley, L. 2007, ApJ, 662, L111

Majewski, S. R., Skrustkie, M. F., Weinberg, M. D., \& Ostheimer, J. C. 2003, ApJ, 599, 1082

Mastropietro, C., Moore, B., Mayer, L., Wadsley, J. \& Stadel, J. 2005, MNRAS, 363, 509

McBride, V. A., Bird, A. J., Cole, M. J., Townsend, L. J., Corbet, R. H. D., \& Haberl, F. 2010, MNRAS, 403, 709

Monelli, M., Carrera, R., Gallart, C., Meschin, I., Aparicio, A., Hidalgo, S. L., Bono, G., Stetson, P. B., \& Walker, A. R. 2011, in "A Universe of Dwarf Galaxies" eds. M. Koleva, Ph. Prugniel, and I. Vauglin, EAS Publication Series, 48, 43

Nidever, D., Majewski, S. R., Burton, W. B., \& Nigra, L. 2010 , ApJ, 723, 1618

Olsen, K. A. G., Zaritsky, D., Blum, R. D., Boyer, M. L., \& Gordon, K. D. 2011, ApJ, 737, 29 (O11)

Piatek, S., Pryor, C. \& Olszewski, E. W. 2008, AJ, 135, 1024

Putman, M., E., Staveley-Smith, L., Freeman, K. C., Gibson, B. K., \& Barnes, D. G. 2003, ApJ, 586, 170

Reed, B. C. 2003, AJ, 125, 2531

Reed, B. C. 2005, AJ, 130, 1652

Robin, A. C., Reylé., C., Derrière, S., \& Picaud, S. 2003, A\&A, 409, 523

Rolleston, W. R. J., Hambly, N. C., Keenan, F. P., Dufton, P. L., \& Saffer, R. A. 1999, A\&A, 347, 69

Schlegel, D. J., Finkbeiner, D. P. \& Davis, M. 1998, ApJ, 500, 525

Sheth, K., Vogel, S. N., Regan, M. W., Teuben, P. J., harris, A. I., \& Thornley, M. D. 2002, AJ, 124, 2581

Skrutskie, M. F. et al. 2006, AJ, 131, 1163

Subramanian, S. \& Subramanian, A. 2012, ApJ, 744, 128

Szczygiel, D. M., Pojmanski, G., \& Pilecki, B. 2009, Acta Astronomica, 59, 137

Udalski, A., Soszynski, I., Szymansli, M. K., Kubiak, M. Pietrzynski, G., Wyrzykowski, L., Szewczyk, O., Ulaczyk, K., \& Poleski, R. 2008, Acta Astronomica 58, 89

Vallée, J. 2005, AJ, 130, 569

van der Marel, R. P. \& Cioni, M.-R. L. 2001, AJ, 122, 1807

van der Marel, R. P., Alves, D. R., Hardy, E. \& Suntzeff, N. B. 2002, AJ, 124, 2639

Vennes, S., Kawaka, A., \& Németh, P. 2010, MNRAS, 410, 2095

Véron-Cetty, M. -P., \& Véron, P. 2006, A\&A, 455, 773

Vieira, K., Girard, T. M., van Altena, W. F., Zacharias, N., CasettiDinescu, D. I., Korchagin, V. I., Platais, I., Monet. D. G., López, C. E., Herrera, D., \& Castillo, D. J. 2010, AJ, 140, 1934 (V10) 